\title{
ARTICLE
}

\section{Human ESC-derived expandable hepatic organoids enable therapeutic liver repopulation and pathophysiological modeling of alcoholic liver injury}

\author{
Shuyong Wang ${ }^{1,2}$, Xuan Wang ${ }^{1,3}$, Zuolong Tan ${ }^{1}$, Yuxin Su ${ }^{1}$, Juan Liu ${ }^{1,4}$, Mingyang Chang ${ }^{1}$, Fang Yan ${ }^{1}$, Jie Chen ${ }^{5}$, Tao Chen ${ }^{5}$,
} Chuanjiang $\mathrm{Li}^{6}$, Jie $\mathrm{Hu}^{3}$ and Yunfang Wang ${ }^{1,4}$

We report the generation of human ESC-derived, expandable hepatic organoids (hEHOs) using our newly established method with wholly defined (serum-free, feeder free) media. The hEHOs stably maintain phenotypic features of bipotential liver stem/progenitor cells that can differentiate into functional hepatocytes or cholangiocytes. The hEHOs can expand for 20 passages enabling large scale expansion to cell numbers requisite for industry or clinical programs. The cells from hEHOs display remarkable repopulation capacity in injured livers of FRG mice following transplantation, and they differentiate in vivo into mature hepatocytes. If implanted into the epididymal fat pads of immune-deficient mice, they do not generate non-hepatic lineages and have no tendency to form teratomas. We further develop a derivative model by incorporating human fetal liver mesenchymal cells (hFLMCs) into the hEHOs, referred to as hFLMC/hEHO, which can model alcoholic liver disease-associated pathophysiologic changes, including oxidative stress generation, steatosis, inflammatory mediators release and fibrosis, under ethanol treatment. Our work demonstrates that the hEHOs have considerable potential to be a novel, ex vivo pathophysiological model for studying alcoholic liver disease as well as a promising cellular source for treating human liver diseases.

Cell Research (2019) 29:1009-1026; https://doi.org/10.1038/s41422-019-0242-8

\section{INTRODUCTION}

Organoid technology has emerged as a powerful and revolutionary strategy enabling the studies of human development and diseases, as well as the applications in drug discovery and clinical treatments. ${ }^{1}$ Various human organoids have been established from the tissues of gut, ${ }^{2}$ retina, ${ }^{3}$ brain, ${ }^{4}$ kidney, ${ }^{5}$ pancreas, ${ }^{6}$ lung, ${ }^{7}$ and even some tumors, ${ }^{8}$ enabling novel models for biomedical researches. Recently, hepatic organoids were also successfully established from primary liver tissues under specially designed culture conditions. ${ }^{9,10}$ The cells inside these tissue-derived hepatic organoids could be expanded and could engraft into the damaged liver upon transplantation. Moreover, they were able to recapitulate the pathogenesis of some genetically inherited liver disorders. ${ }^{9}$ These previous findings suggested applications with considerable potential for liver disease modeling using hepatic organoids as well as for transplantation therapy.

Due to the limitations in the availability and throughput of human primary liver tissues from either healthy or diseased liver donors, the robust production of such valuable hepatic organoids needed for large-scale applications has remained challenging. Human pluripotent stem cells (hPSCs) can self-renew indefinitely and can differentiate into all cell types in the human body, thus offering an alternative to the use of primary tissues for generating the desired hepatic organoids. The spontaneous aggregation of hepatocytes in 3D suspension was first used to form hepatic organoids to improve functionality of hPSC-derived hepatocytelike cells, ${ }^{11-13}$ but the expansion potential of the resulting cells was negligible. Furthermore, by use of co-culture of hepatic endoderm cells from hPSCs with liver-associated, nonparenchymal cell types, the hepatic organoids named as liver buds were generated, ${ }^{14}$ which recapitulated fetal liver features in vitro and exhibited mature hepatic functions after ectopic transplantation. Nevertheless, this model system is not proliferative and has the limitation of scalability. Most recently, it was reported that multilayered organoids capable of modeling hepatic genetic diseases have been generated from hPSCs using a method completing the whole differentiation process in $3 \mathrm{D} .{ }^{15}$ However, the exact level of cell expansion for these hepatic organoids from hPSCs was not reported. In addition, the in vivo engraftment and repopulation efficiency of all the previously reported hPSC-derived hepatic organoids after transplantation into the liver has not been studied yet. ${ }^{11-15}$ On the other hand,

\footnotetext{
${ }^{1}$ Tissue Engineering and Regenerative Medicine Lab, Beijing Institute of Health Service and Transfusion Medicine, 100850 Beijing, China; ${ }^{2}$ Army Tuberculosis Prevention and Control Key Laboratory, Beijing Key Laboratory of New Techniques of Tuberculosis Diagnosis and Treatment, Institute of Tuberculosis Research, The 8th Medical Center of Chinese PLA General Hospital, 100091 Beijing, China; ${ }^{3}$ Department of Nursing, Hebei Medical University, 050017 Shijiazhuang, China; ${ }^{4}$ Hepatal-Biliary-Pancreatic Center, Translational Research Center, Beijing Tsinghua Chang Gung Hospital, 102218 Beijing, China; ${ }^{5}$ Department of Hepatobiliary Surgery, Sun Yat-sen Memorial Hospital, Sun Yat-sen University, 510289 Guangzhou, China and ${ }^{6}$ Department of Hepatobiliary Surgery, Nanfang Hospital, Southern Medical University, 510515 Guangzhou, China Correspondence: Yunfang Wang (wangyf1972@gmail.com)

These authors contributed equally: Shuyong Wang, Xuan Wang

These authors are co-senior authors: Jie Hu, Yunfang Wang
}

Received: 19 November 2018 Accepted: 19 September 2019

Published online: 18 October 2019 
1010

besides modeling genetically inherited liver disorders, ${ }^{9,15}$ the potential applications of hepatic organoids have not been studied on modeling the non-genetic human metabolic diseases, especially those with pathophysiological processes.

In this study, we have developed a new method that relies on uniquely defined culture media to make a successful shift from $2 D$ to $3 \mathrm{D}$ cultures for generating human embryonic stem cell-derived expandable hepatic organoids (hEHOs). Our established hEHOs exhibit bipotential traits for lineage restriction into functional hepatocytes and cholangiocytes and also show significant expansion potential for 20 passages. Remarkably, our hEHOs can be re-established from a single cell of dissociated hEHOs and a single cell-derived hEHOs proved to be bipotential, which has not been demonstrated in any previously reported hepatic organoids from hPSCs. Moreover, our hEHOs can efficiently engraft into and repopulate the liver parenchyma of FRG mouse following transplantation. Finally, 3D co-culture hepatic organoids generated with hEHOs and human fetal liver mesenchymal cells (hFLMCs) faithfully recapitulated the aspects of the human alcoholic liver disease (ALD)-associated pathophysiology after ethanol treatment, which might be a powerful in vitro model for mechanism studies, new biomarkers screening and novel treatment targets exploration of ALD.

\section{RESULTS}

The hEHOs were stably expanded after long-term 3D cultures A stepwise differentiation of hESCs into hepatocytes was induced through mimicking embryonic liver development. ${ }^{16-19}$ After 3 days of exposing cells to Activin A with Wnt3A for definitive endoderm (DE) formation, the cells were further treated with BMP4 and FGF2 for another 5 days for their restriction into a hepatic specification (HS) lineage under conditions of monolayer (2D) cultures (Fig. 1a, b). At the end of the induction stage for HS cells, it was confirmed that most of the cells were positive for the well-known hepatic stem/progenitor cell markers including HNF4A, CK19, EPCAM, SOX9, AFP (Supplementary information, Fig. S1). After screening the culture conditions for formation of hepatic organoids, ${ }^{9,20,21}$ they were subjected to treatments with the combination of N2 (N), B27 (B), Nicotinamide (Ni), Gastrin (G), N-Acetylcysteine (Na), EGF (E), Wnt3a (W), R-Spondin 1 (R), A83-01 (A) and Forskolin (F) (NBNiGNaEWRAF). This resulted in the generation of hEHOs. The derived hEHOs initially appeared as hollow spherical structures in approximately 2 days, then rapidly grew into large three-dimensional (3D) organoids within 10 days (Fig. 1c). They were able to expand by passaging at a 1:6-1:8 ratio every 7-9 days for over 20 passages (Fig. 1d). Remarkably, they remained normal with respect to both survival and expansion after freezing and thawing (Fig. 1d). After long term passaging, they still maintained exponential growth with a stable cell doubling time (Fig. 1e). Theoretically, $10^{18} \mathrm{hEHOs}$ could be generated after 20 passages from $10^{4}$ initiating hEHOs, which should be more than enough for the number of cells usually required for large scaled investigations in industry or academia or for transplantation therapy of a single patient. ${ }^{22}$ The karyotype analysis on dissociated cells of hEHOs proved that their chromosome numbers were all normal after more than 4 months in culture (Fig. 1f).

hEHOs showed the phenotypic traits of hepatic stem/progenitor cells

Morphologically, hEHOs were similar to the human adult liver (bile duct tissues)-derived hepatic organoids (hAHOs) ${ }^{9}$ (Supplementary information, Fig. S2a). Next, the gene expression profile of hEHOs was further characterized. To define the developmental stages of hEHOs, transcriptomes were established for several groups, including the hEHOs at both passage 1 and passage 3 , hESCderived foregut stem cells (FSCs), ${ }^{23}$ HS cells, ${ }^{17,24}$ hepatoblasts
$(\mathrm{HB}){ }^{24}$ hAHOs and primary human hepatocytes (PHH). Remarkably, hEHOs showed similarities with HS cells and HB on the genome-wide gene expression profiles (Supplementary information, Fig. S2b). Principal-component analysis further displayed a clear clustering of each of the analyzed groups and the hEHOs (both passage 1 and passage 3 ) showed more similarity to HS cells and $\mathrm{HB}$ but remained distinct from them (Fig. 2a). Euclidean hierarchical cluster analysis revealed that hEHOs were phenotypically between FSCs and two groups of immature hepatic lineages (HS and HB). They were closely related to both HS cells and $\mathrm{HB}$ but strongly distinct from $\mathrm{PHH}$ (Fig. 2b); this was also reflected by the results of Pearson correlation coefficient (hEHOs versus HS: $r=0.83-0.85$, hEHOs versus HB: $r=0.83-0.85$, hEHOs versus hAHOs: $r=0.80-0.83$, hEHOs versus FSCs: $r=0.80-0.82$, and hEHOs versus PHH: $r=0.75-0.76$ ) (Supplementary information, Fig. S2c).

As expected, significant expression of mature hepatocytes markers (CYP3A4, CYP1A2, CYP2E1, CYP2A6, CYP2C8, CYP2C9 and CYP2D6) was limited in $\mathrm{PHH}$, while the expression of specific markers of foregut endoderm (SOX17, SOX2, KDR and CER1) was up-regulated in FSCs (Fig. 2c). Although hEHOs and hAHOs shared similarities in morphology and in expression of some hepatic stem/progenitor-specific genes (HNF4A, TBX3, CEBPA, CEBPB, SOX9, CK19 and EPCAM) at the transcriptional level (Supplementary information, Fig. S3a), they exhibited some differences. The hEHOs showed up-regulation of several transcription factors and regulators of early liver development (PROX1, ONECUT2, SOX9, FGFR4, FOXA1 and FOXA3), while the up-regulated components for Wnt signaling (LGR5, ZNRF3, RNF43, AXIN2, CD44, DKK1, WNT7A and WNT7B) were observed in hAHOs (Fig. 2c). On the other hand, results of immunofluorescence assays revealed that the hEHOs were negative for the pluripotency markers (OCT4 and SOX2) but were positive for hepatic stem/progenitor markers (SOX9, EPCAM, CK19, HNF4A, TBX3 and AFP). Furthermore, results of Ki67 immunofluorescence assay suggested that cell proliferation was significant in hEHOs (Fig. 2d). Accordingly, the heterogeneity of hEHOs was also analyzed by flow cytometry. CK19, EPCAM, HNF4A, SOX9, and TBX3 were detectable in almost all of the cells of hEHOs, whereas AFP was detectable only in $18.1 \%$ of the cell populations (Supplementary information, Fig. S3c). The hepatic stem cell/progenitor markers remained relatively stable in both early and late passaging stages of hEHOs (Supplementary information, Fig. S3a). Together, the above results demonstrated that hEHOs closely resembled the cells at nascent stages of the hepatic lineage, reflected by the facts that they were endowed with hepatic stem/progenitor cell phenotypes.

hEHOs were restricted to hepatic lineages in vivo and had no oncogenic potential

The hEHOs were further investigated for their capacity of spontaneous lineage restriction into hepatic derivatives. The hEHOs were transplanted into the epididymal fat pads of nonobese, diabetic, severe combined immune-deficiency mice, and the transplanted hEHOs were analyzed after 4-10 weeks of growth under in vivo condition. The transplanted hEHOs reproducibly gave rise to the ductal epithelial structures (Fig. 3a, b). Results of immunostaining assays revealed that the grafts of hEHOs showed heterogeneity associated with spontaneous differentiation. More specifically, some of the cells expressed mature hepatocyte markers (TF, ASS1, CYP3A4, AAT, ALB, and CK18); some of them were positive for fetal cholangiocyte markers (SOX9 and CK19); and some were $\mathrm{ALB}^{+}$CK19 ${ }^{+}$hepatoblasts (Fig. 3c, Supplementary information, Fig. S4a). Very few cells were present with phenotypic traits consistent with mature cholangiocytes, which expressed CK7 but weakly expressed or did not express SOX9 (Supplementary information, Fig. S4a). Results of coimmunostaining assays combining human-specific anti-Mitochondrial antibody (Mito) with antibodies against CK19, ALB and SOX9 
a
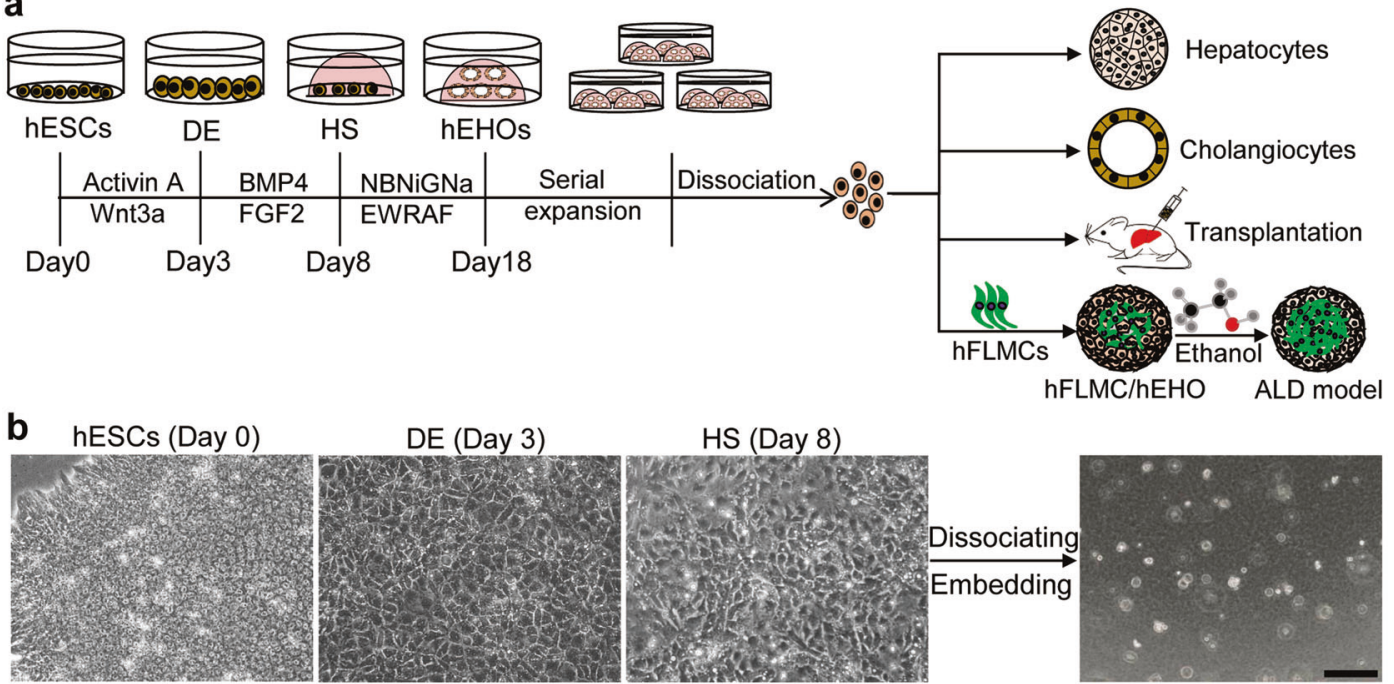

HS (Day 8)

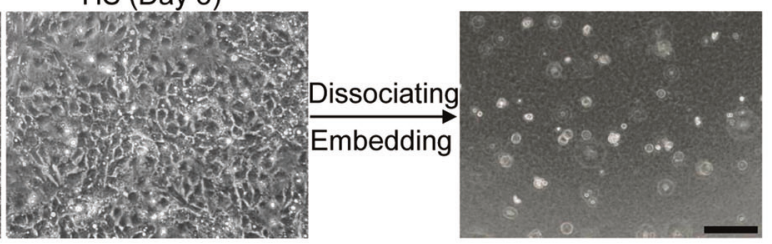

C

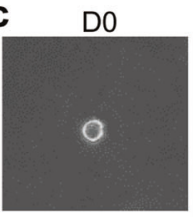

D2

D5
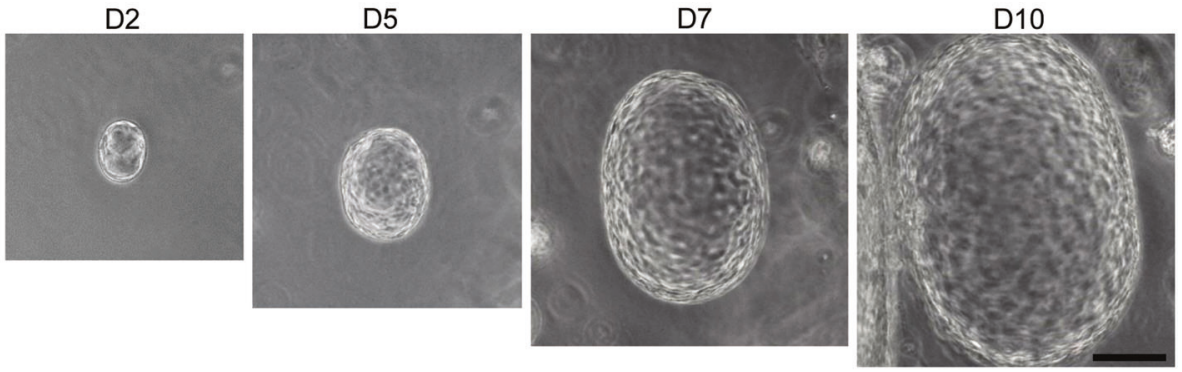

d
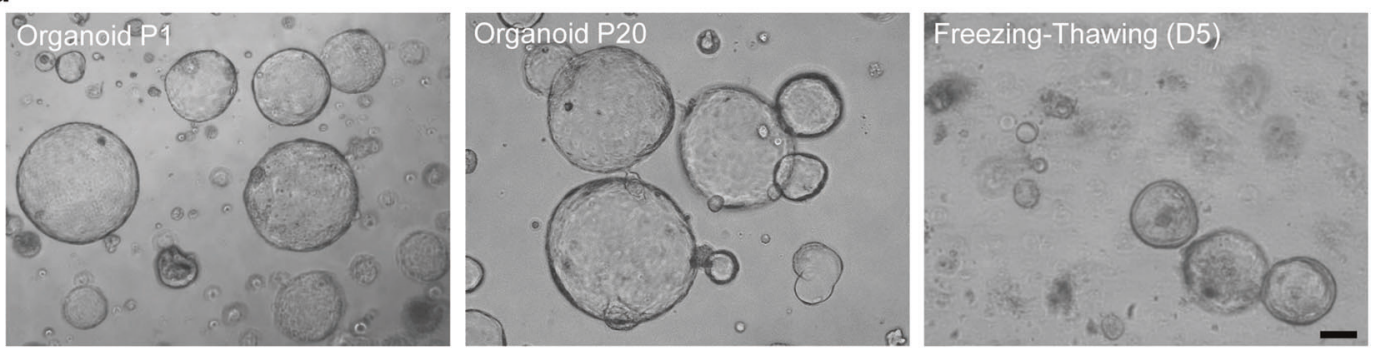

e

\section{P1-P3}

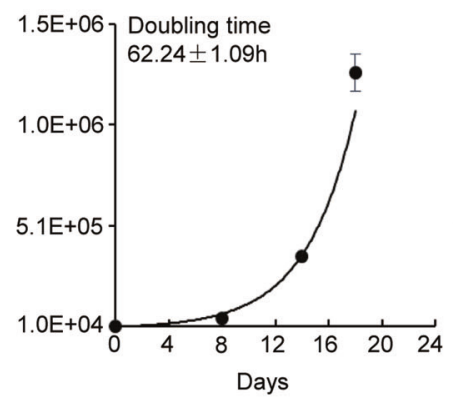

P13-P15

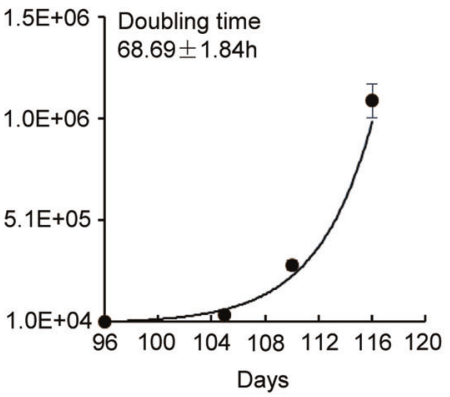

f

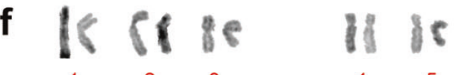

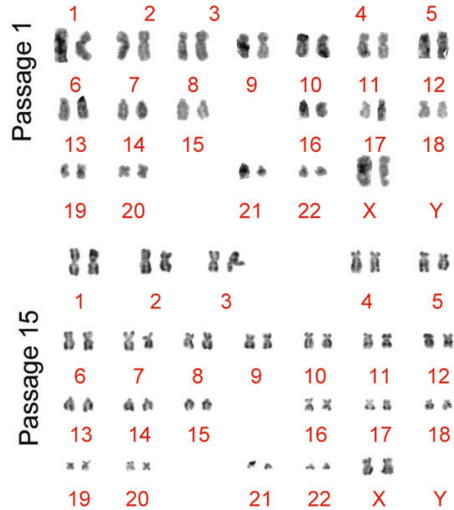

Fig. 1 Establishment of hEHOs culture system. a Schematic of the experimental protocol. $\mathbf{b}$ Phase-contrast microscopic images of the cells at different stages. c Representative serial images of hEHOs cultures growing at the indicated time points. $\mathbf{d}$ Representative images at indicated passage numbers and the morphology of post-thawed hEHOs on day 5. e In vitro growth curves were analyzed at early and late passages of hEHOs. f Representative karyotypes of a cell in Passage 1 and 15 with normal chromosome number $n=46$. Scale bar: $100 \mu$ m. Data are presented as mean \pm SD ( $n=3$ per time point in e) 
a

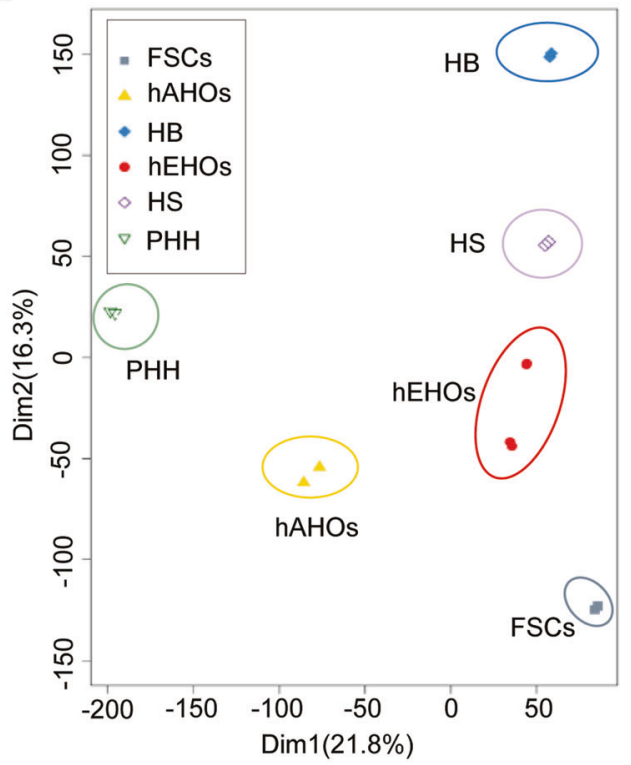

b

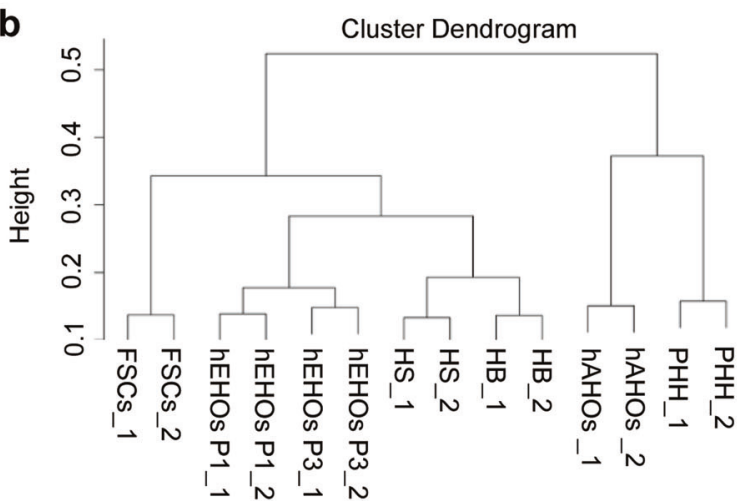

C

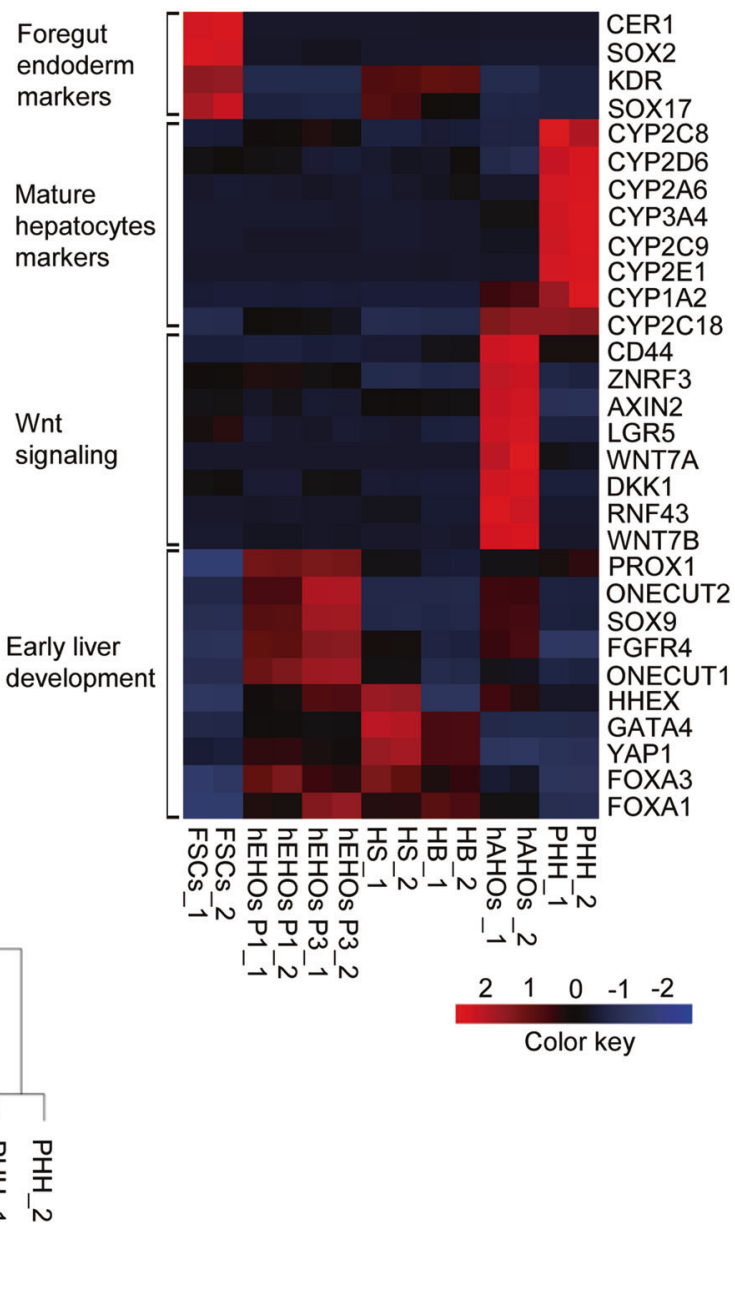

d

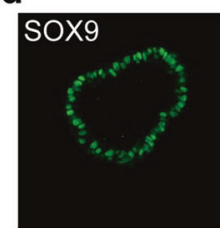

ALB

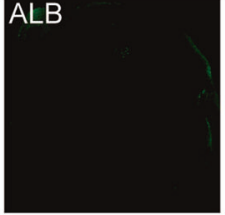

OCT4

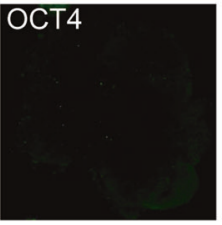

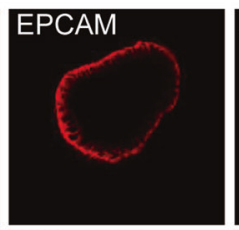
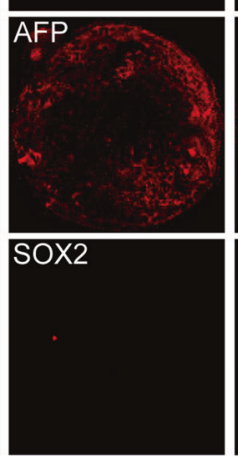
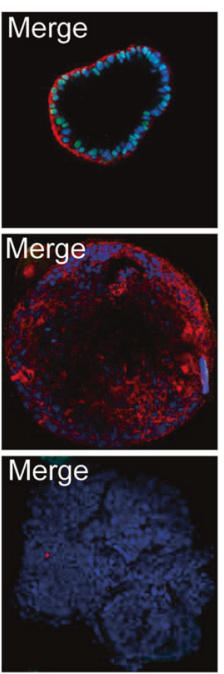
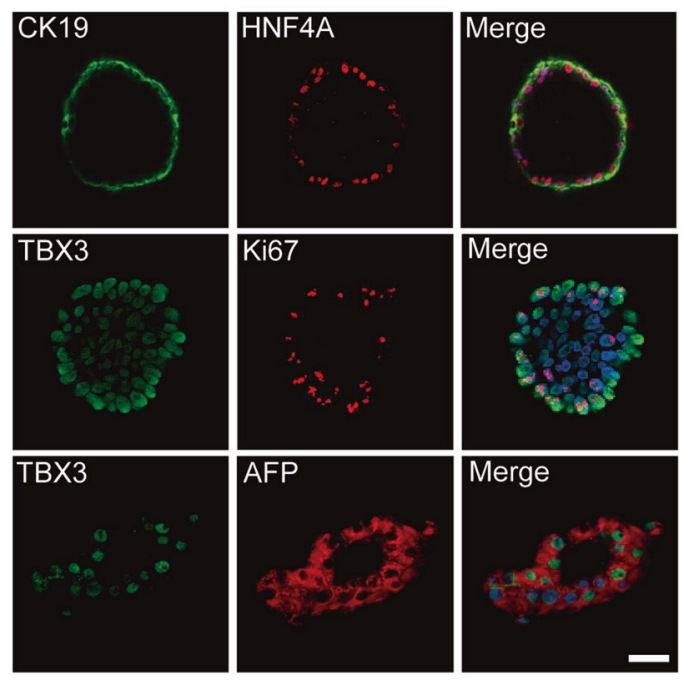

Fig. 2 hEHOs are endowed with properties of hepatic stem/progenitor cells. a Principal component analysis of PHH, hAHOs, FSCs, HS cells, HB, and hEHOs at passage 1 and passage 3 based on global gene expression profiles. b Euclidean hierarchical clustering of PHH, hAHOs, FSCs, HS cells, $\mathrm{HB}$, and hEHOs at passage 1 and passage 3 using differentially expressed genes. c Heatmap showing the expression of selected genes in PHH, hAHOs, FSCs, HS cells, HB, and hEHOs at passage 1 and passage 3. d Immunostaining of hEHOs with SOX9, CK19, EPCAM, HNF4A, AFP, ALB, TBX3 and Ki67. Scale bar: $50 \mu \mathrm{m}$ (d). hEHOs-P1, hEHOs of passage 1; hEHOs-P3, hEHOs of passage 3 
a

b
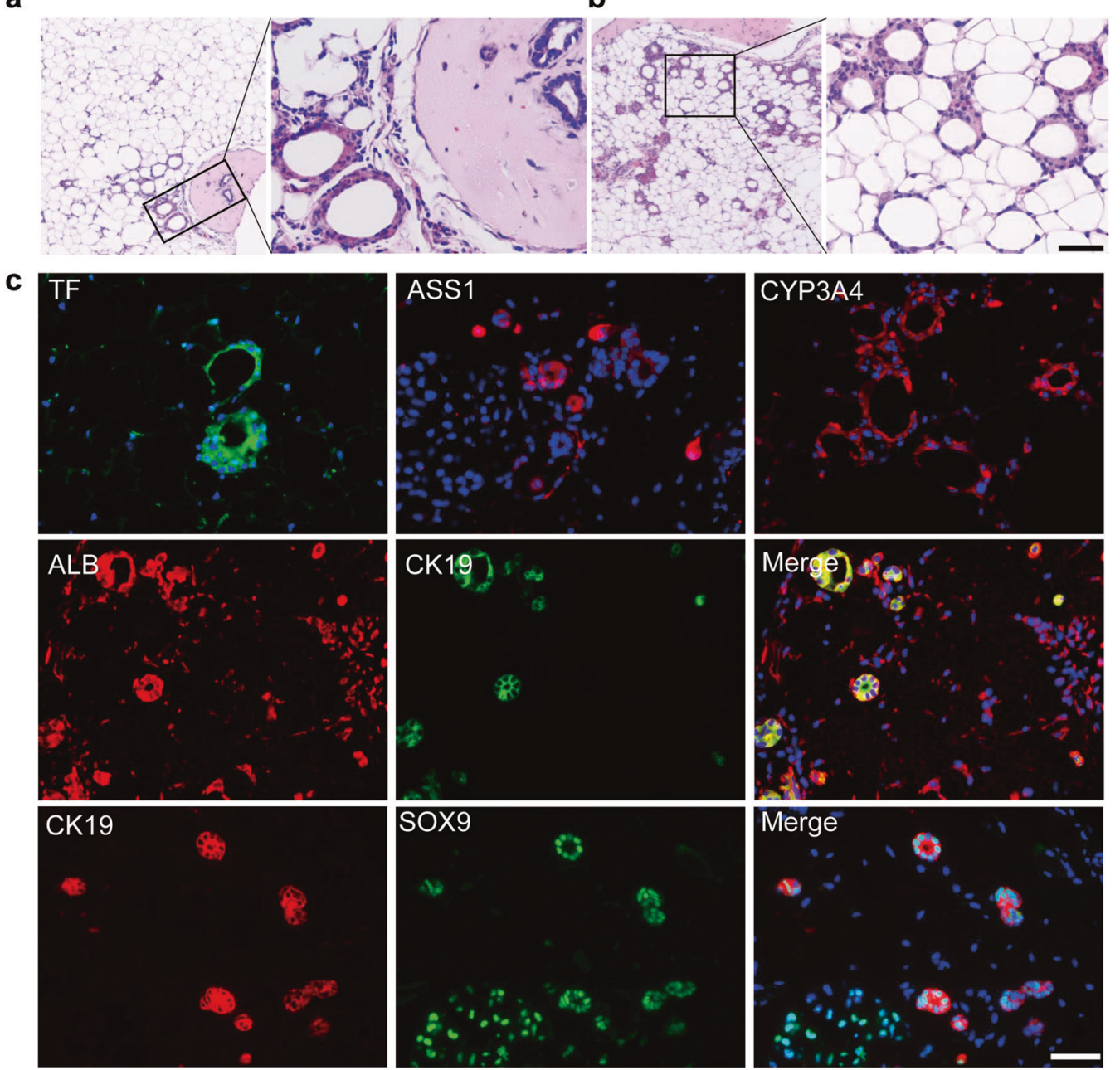

d
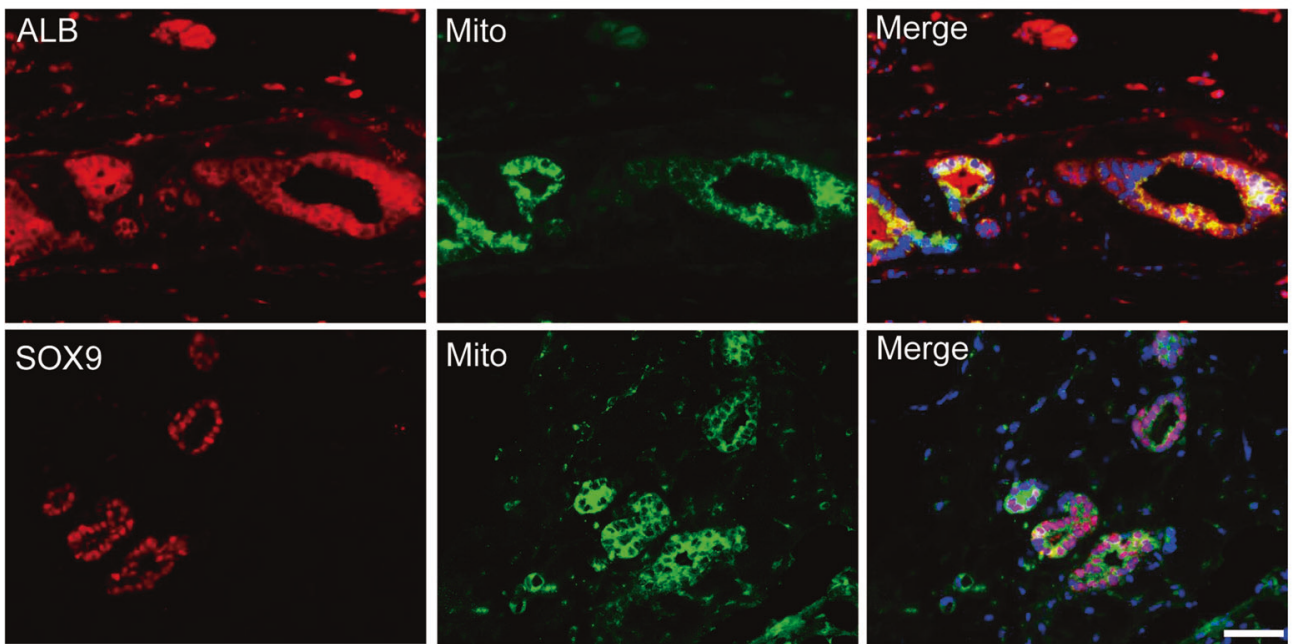

Fig. 3 Hepatic lineage-restricted potential of hEHOs in vivo. a, b Sections of hEHO-derived grafts were stained with Hematoxylin and Eosin. Representative ductal structures in Matrigel plugs (a) and in adjacent fat tissue (b) are shown. c Immunofluorescence staining for hepatic markers (TF, ASS1, CYP3A4, and ALB), hepatoblast markers (ALB and CK19), cholangiocyte markers (CK19 and SOX9) in the grafts. d Human cell mitochondria co-immunostaining with SOX9, ALB in the grafts. Scale bar: $50 \mu \mathrm{m}$. Mito, human anti-mitochondrial antibody

demonstrated that the hepatic structures were derived from the transplanted hEHO cells (Fig. 3d, Supplementary information, Fig. S4a). Remarkably, unlike FSCs which could differentiate into endodermal derivatives, including hepatic and pancreatic lineages, ${ }^{23}$ neither intestinal (CDX2 and MUC2), gastric (GASTRIN and MUC6), nor pancreatic (PDX1 and PRO-INSULIN) markers were observed in hEHO-derived cells (Supplementary information, Fig. S4b-d), suggesting that hEHOs could not differentiate into cell types other than hepatic lineages after transplantation. Together, these data demonstrated that the hEHOs were restricted to the hepatic lineage and had no oncogenic potential in all analyzed mice with transplanted hEHOs $(n=6)$. 
Cells of hEHOs could differentiate into functional hepatocytes in vitro

Based on the findings of bipotential hepatic lineages for transplanted hEHOs in vivo, cells of hEHOs were further investigated for the potential to differentiate into functional hepatocytes in vitro. Previous studies have demonstrated that 3D culture contributed to achieving better hepatic differentiation of hPSCs. ${ }^{11-13}$ Through modification of the methods from previous reports, ${ }^{9,11,25}$ the 3D culture condition for hEHOs' differentiation into hepatocytes was established. Within 15 days, the dissociated hEHOs in suspension differentiated and gradually formed solid aggregates with smooth edges that expanded to approximately $100 \sim 150 \mu \mathrm{m}$ in diameter (Fig. 4a). The hepatic specific genes (HNF4A, AAT, CK18, TAT, TF, G6PC, and $A L B)$, genes encoding urea cycle enzymes (CPS1, OTC, ASS1, ASL and $A R G 1)$, and drug metabolism genes (CAR, FXR, PXR, CYP2C9, CYP2E1 and CYP3A4) were all up-regulated in hEHO-derived hepatocytes (hEHO-Heps) when compared with those in undifferentiated hEHOs and hESC-derived 2D hepatocytes ${ }^{17,18}$ (hESC-Heps (2D)), and the expression of some of these genes closely resembled that in primary human hepatocytes (PHH) (Fig. 4b, Supplementary information, Fig. S5a). The capacity of hepatic differentiation of hEHOs was stably maintained during passaging of the cells (Fig. 4b, Supplementary information, Fig. S5a). Results of immunostaining analyses also revealed that $\mathrm{hEHO}$-Heps expressed mature hepatic markers (ALB, AAT, TF, HNF4A, CK18, CYP3A4, MRP2 and CPS1) (Fig. 4c, Supplementary information, Fig. S5b). Flow cytometric analyses revealed that a relatively high efficiency of hepatic differentiation was achieved; approximately $93 \%$ of the differentiated cells were $\mathrm{ALB}^{+}$, and most of the cells (92.7\%) were positive for expression of ALB and AAT $\left(\mathrm{ALB}^{+} \mathrm{AAT}^{+}\right)$. About $20 \%$ of the cells were $\mathrm{ALB}^{+} \mathrm{AFP}^{+}$, traits similar to those of fetal hepatocytes (Fig. $4 \mathrm{~d}$ ). Additionally, ultrastructural analyses with electron microscopy showed typical hepatocyte organelles; the hEHO-Heps were replete with mitochondria, endoplasmic reticulum, lipid droplet and glycogen granules (Supplementary information, Fig. S5c). Interestingly, the bile canaliculi were observed in the intercellular space of adjacent cells demonstrating the establishment of functional epithelial polarization that was essential to hepatocyte function in 3D aggregates (Fig. 4e). Levels of albumin secretion, urea production and CYP3A4 metabolic activities in hEHO-Heps were significantly increased when compared with those in the undifferentiated hEHOs and hESC-Heps (2D), but were still not as high as the levels found in the PHH (Fig. 4f), suggesting the necessity for further improvement in induction of mature hepatic phenotypic traits. In addition, other important parameters of hepatocyte-specific functions were also found in hEHO-Heps, such as the uptake and release of indocyanine green (ICG), as well as glycogen storage (PAS) at significantly high levels (Fig. $4 \mathrm{~g}-\mathrm{i}$ ). Furthermore, both formation of bile canaliculi and function of the transporter were evaluated in hEHO-Heps after exposure to CDFDA, a dye hydrolyzed to fluorescent CDF, which can be secreted by MRP2. Intercellular distribution of bright CDF fluorescence inside of the aggregates further demonstrated that hEHO-Heps were functionally polarized hepatocytes (Fig. 4j). Taken together, all of the above results clearly indicated that hEHOs have the capacity under in vitro 3D culture condition to become cells with many of the traits of mature hepatocytes.

Cells of hEHOs could differentiate into mature cholangiocytes in vitro

Next, hEHOs were assessed for the capacity to differentiate into mature cholangiocytes under in vitro 3D culture condition that was modified from those in previous reports. ${ }^{26,27}$ Results of morphological analyses revealed that the hollow ductal structures gradually formed and expanded over 10 days (Supplementary information, Fig. S6a). Gene expression analyses revealed that all of the cholangiocyte markers (CK19, EPCAM, CFTR, GGT and AQP1), transporters (ASBT, FABP6 and MRP3) and cholangiocyteassociated transcription factors (SOX9, HES1, HNF6, and HNF1B) were up-regulated in hEHO-derived cholangiocytes (hEHO-Clcs), when compared with those markers in undifferentiated hEHOs. Notably, the levels of some gene expression found in these structures were close to those detected in primary cholangiocytes (PCs) (Supplementary information, Fig. S6b). By contrast, hepatocyte-associated markers (TBX3, HNF4A and AFP) were down-regulated after differentiation into cholangiocytes (Supplementary information, Fig. S6b). Results of immunostaining analyses showed that $\mathrm{hEHO}-\mathrm{Clcs}$ expressed mature biliary markers (CK7, CK19, CFTR and AQP1) but not the hepatic marker (ALB) (Supplementary information, Fig. S6c). Results also indicated that F-actin localized in the apical domains of the lumen, while $\beta$ catenin distributed along basolateral domains. In addition, Ecadherin localized at cell-cell contacts, which showed the polarity of the epithelial cells inside the ductal structures (Supplementary information, Fig. S6c). A physiological function of cholangiocytes is the regulation of the bile composition by excretion of various ions, solutes, and water. To assess the secretory functions, the hEHOClcs were evaluated for the capacity to efflux Rhodamine 123, a dye used to measure activity of the multidrug resistance protein 1 (MDR1) transporter in normal cholangiocytes. Results showed that the cystic structures transported dye to the luminal space (Supplementary information, Fig. S6d). Moreover, accumulation of Rhodamine could not be found in the lumen when Verapamil (Ver) was applied as an inhibitor of MDR1 at $10 \mu \mathrm{M}$, which confirmed that the dye movement reflected active transportation through MDR1 in the hEHO-Clcs (Supplementary information, Fig. S6d). Furthermore, alkaline phosphatase (ALP) activities in $\mathrm{hEHO}-\mathrm{Clcs}$ were also observed, indicating that the functional phosphatase was expressed specifically (Supplementary information, Fig. S6e). Additionally, hEHO-Clcs' responses to acetylcholine and ATP stimuli by increasing intracellular calcium were also confirmed (Supplementary information, Fig. S6f). In contrast, undifferentiated hEHOs were negative for CK7 and AQP1 with weak expression of CFTR, and showed weak or negligible transportation of Rhodamine and ALP staining (Supplementary information, Fig. S7a-c). Thus, these findings demonstrated that $\mathrm{hEHO}-\mathrm{Clcs}$ in cysts acquired both structural and functional characteristics of mature cholangiocytes.

Bipotentiality of hEHOs was confirmed by their capacity for clonal differentiation

In order to further prove the bipotential differentiation capacity, the hEHOs were dissociated into single cells in suspension, and were processed for FACS-based single-cell sorting (Fig. 5a). Each single cell was sorted directly into organoid formation medium containing 5-10\% Matrigel in non-tissue culture-treated 96-well plates at a density of 1 cell/well (Fig. 5b). Each single cell gradually expanded and formed a hollow sphere (Fig. 5c). After expansion of a single cell-derived organoid for several passages, the cells were collected and subsequently placed into the media for hepatocytes versus cholangiocytes differentiation using the same methods described above. Remarkably, the dissociated single cell-derived organoids cultured under condition tailored for hepatocytes differentiation generated cells that expressed hepatic markers (ALB, AAT, CK18, HNF4A and AFP) and performed hepatocytespecific functions (Fig. 5d, e). When dissociated organoids cultured in medium tailored for cholangiocytes differentiation, the generated cells expressed cholangiocyte markers (CK7, SOX9, CFTR and AQP1) and acquired functional characteristics of mature cholangiocytes (Fig. 5f, g). Thus, these data strongly proved the bipotentiality of the clonogenic hEHOs.

hEHOs efficiently engrafted and repopulated the damaged livers of mice after transplantation

To examine whether the hEHOs could be functionally and stably integrated into the liver parenchyma, they were dissociated into single cells and directly transplanted into the liver of $\mathrm{Fah}^{-1-}$ / 
a
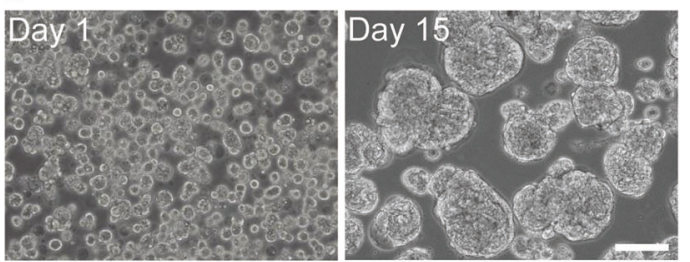

C
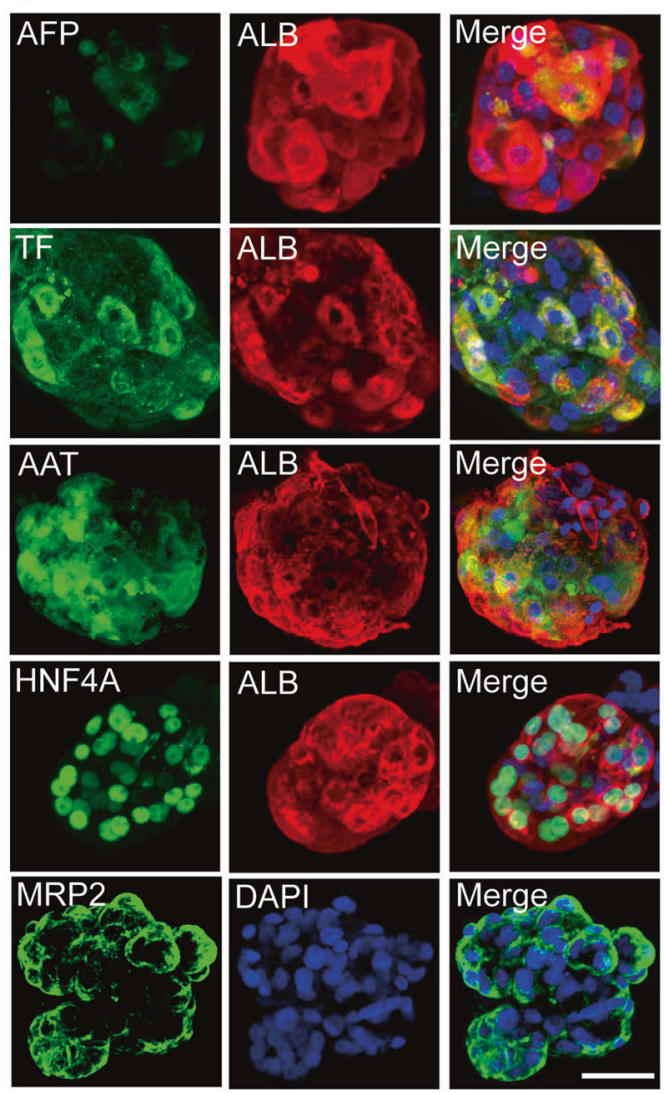

b
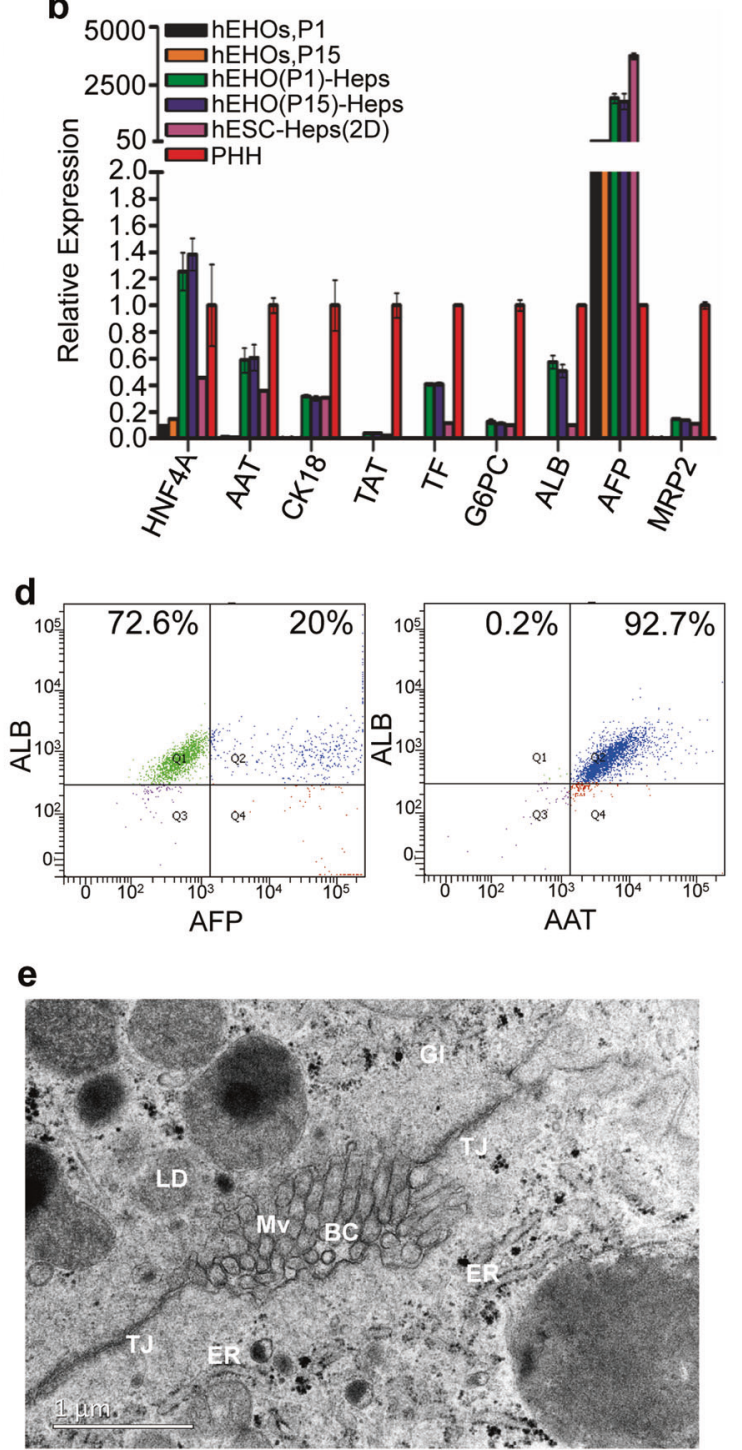

g ICG-uptake

h ICG-excretion
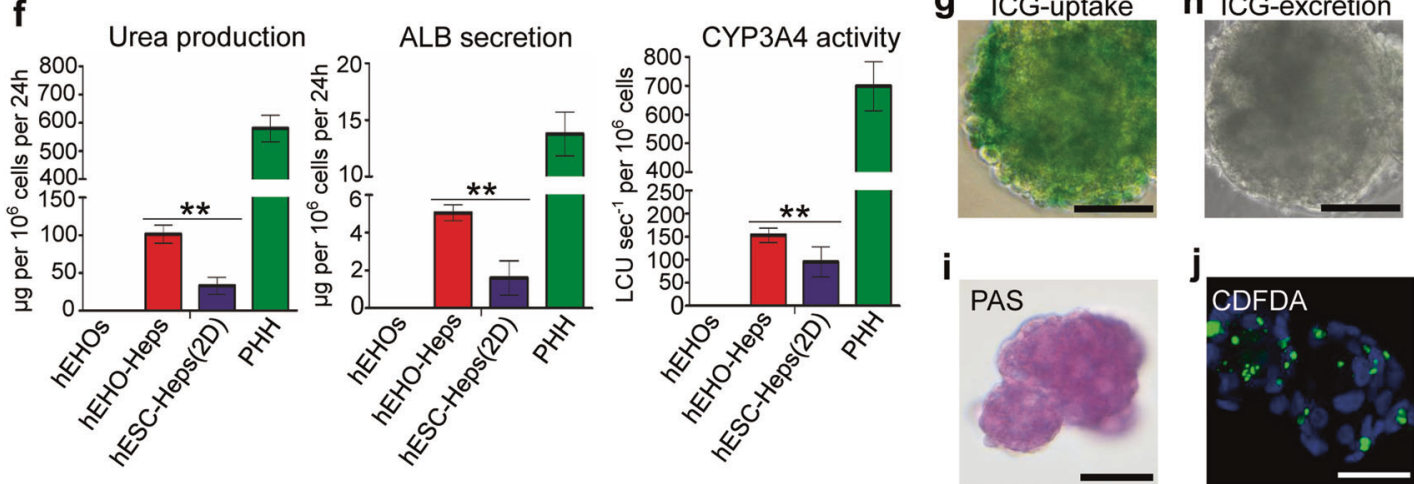

Fig. 4 Cells of hEHOs can differentiate to mature hepatocytes in vitro. a Morphology of hEHO-Heps at indicated times. $\mathbf{b}$ qRT-PCR of hepaticlineage markers in hEHO(P1)-Heps, hEHO(P15)-Heps, undifferentiated hEHOs, hESC-Heps (2D) and PHH. The gene expression levels in PHH were normalized to 1 . Results are presented as mean \pm SD from 3 replicates from three independent repeated experiments. $\mathbf{c}$ Immunostaining

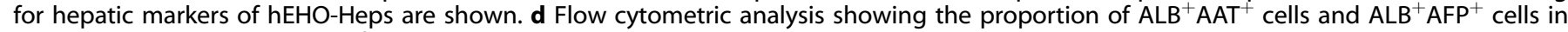
hEHO-Heps. e Ultrastructure of hEHO-Heps showed the intercellular canaliculi. LD lipid droplet, TJ tight junction, Mv microvilli, BC bile canaliculi, Gl glycogen, ER endoplasmic reticulum. $\mathbf{f}$ Representative assays of hepatic functions in hEHO-Heps. Functional characterization of hEHO-Heps. ICG uptake (g) and release (h), PAS (i), CDFDA staining (j). Data are presented as mean \pm SD $(n=3$ per group in f). **P<0.01. Scale bar: $100 \mu \mathrm{m}(\mathbf{a}, \mathbf{g}-\mathbf{i}), 50 \mu \mathrm{m}(\mathbf{c}, \mathbf{j})$. hEHO(P1)-Heps, hEHOs at passage 1-derived hepatocytes; hEHO(P15)-Heps, hEHOs at passage 15-derived hepatocytes 
a

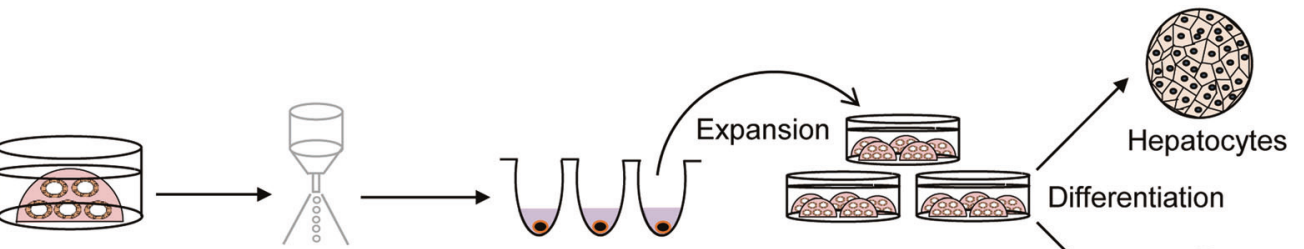

hEHOS

Single cell sorting

Organoid formation

b

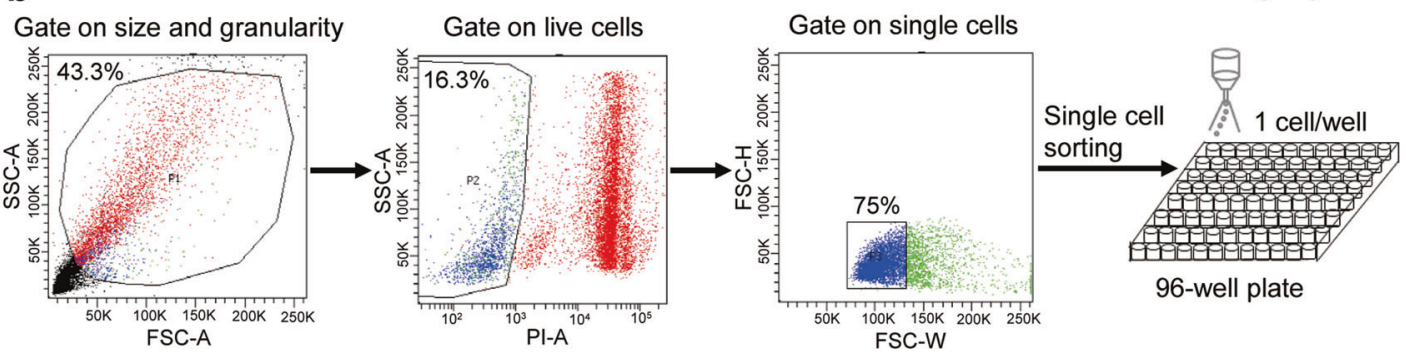

C

D0

D2

D4

D6

D8

D10

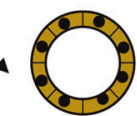

Cholangiocytes

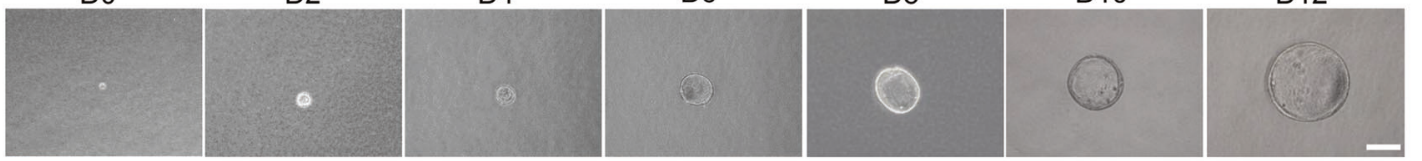

d
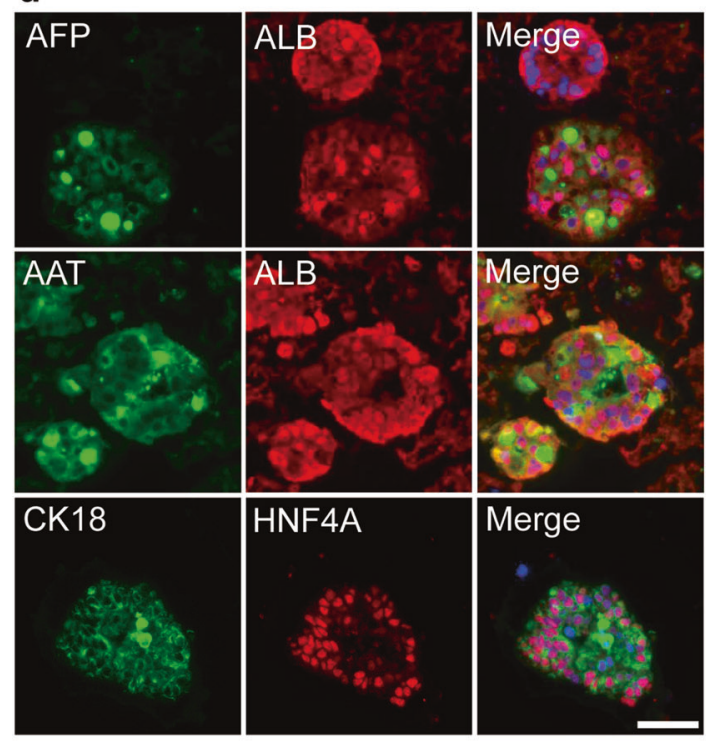

f
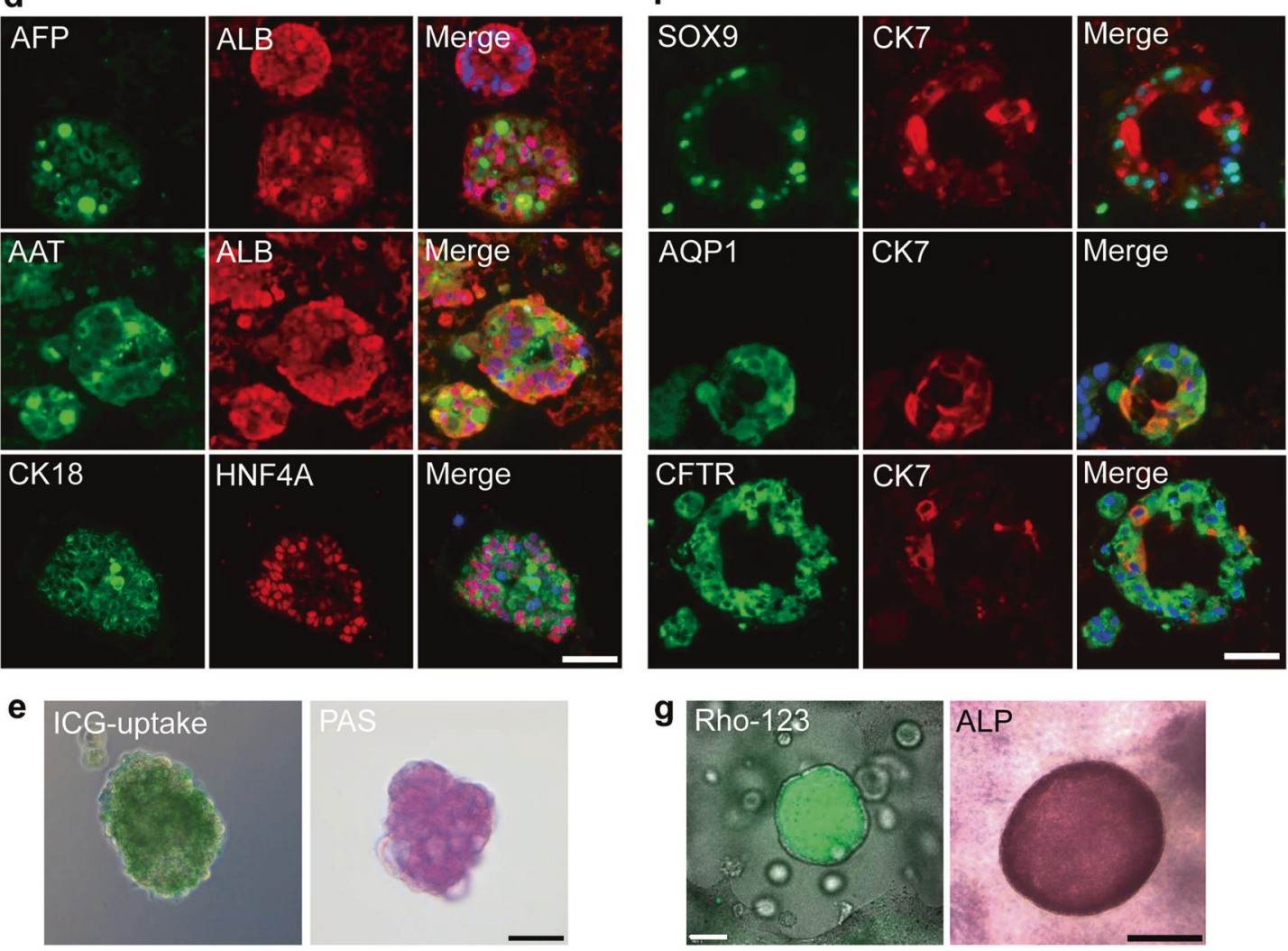

Fig. 5 Single-cell derived hEHOs are bipotential. a Schematic of the experimental strategy for the analysis of the bipotentiality of hEHOs. b FACS-based single-cell sorting of the dissociated cells from hEHOs. c Representative time-lapse imaging for the growth of hEHOs from a single-cell (day 0 [D0] to D12). Differentiation of single-cell derived hEHOs were found to generate cells expressing hepatic markers (ALB, AFP, AAT, CK18, HNF4A) (d), and performing liver functions (e). Differentiation of single-cell derived hEHOs were found to generate cells expressing cholangiocyte markers (CK7,CFTR,AQP1,SOX9) (f), and performing bile-duct associated functions (g). Scale bar: $100 \mu \mathrm{m}(\mathbf{c}, \mathbf{e}, \mathbf{g}), 50 \mu \mathrm{m}(\mathbf{d}, \mathbf{f})$ 
Rag2 $2^{-1-} / 112 \mathrm{rg}^{-1-}$ (FRG) mice usually used as a robust model to characterize in vivo liver engraftment during transplantation assays. $^{28-30}$ All of the FRG mice without cell transplantation died within 5 weeks (Fig. 6a). In contrast, 4 of the 6 FRG mice transplanted with hEHOs survived more than 3 months (Fig. 6a), similar to the survival rate of FRG mice transplanted with PHH as a control. At 4 weeks after transplantation, the early loss of body weight in hEHOs-transplanted mice resolved, and body weight remained stable thereafter, suggesting that transplanted hEHOs gradually matured and restored liver functions in the FRG mice (Fig. 6b). Accordingly, the significant reduction in serum markers of hepatocyte injury, such as alanine aminotransferase (ALT) and aspartate aminotransferase (AST), further demonstrated hepatic maturation of hEHOs and recovery of liver functions (Fig. $6 \mathrm{c}$ ). The secretion level of human albumin in collected serum samples of mice transplanted with hEHOs was around $102 \mu \mathrm{g} / \mathrm{mL}$ on average at 3 months (Fig. 6d). To analyze the repopulation efficiency after transplantation of hEHOs, liver samples were harvested at 3 months after transplantation. Immunohistochemical staining of $\mathrm{FAH}$ showed that hEHO-derived hepatocytes repopulated 20\% $\pm 5.6 \%$ of the liver parenchyma in the surviving mice (Fig. 6e). The repopulation was further proved by positive staining of humanspecific ALB (hALB) and Hep Par-1 (Fig. 6f, Supplementary information, Fig. S8a, b). The hALB-positive cells were also positive for cytokeratin 18 (CK18), a marker of liver function, in the liver parenchyma (Fig. $6 \mathrm{~g}$ ). In addition, the histological distribution of hEHO-derived hepatocytes in vivo was more precisely analyzed with respect to liver zonation. ${ }^{31}$ Co-immunostainings of hALB and other functional liver markers demonstrated that the engrafted cells from hEHOs existed around central veins (GS and CYP2E1 positive), as well as around portal areas (ASS1 positive) (Fig. 6h, Supplementary information, Fig. S8c). Some of the hALB-positive hepatocytes were also found positive for Ki67, the marker of cell proliferation, which confirmed that the repopulating cells demonstrated significant proliferative activity in the liver parenchyma of recipients (Supplementary information, Fig. S8d). Together, all of the above results strongly support that hEHOs have a remarkable capacity of engraftment, maturation in vivo and repopulating the injured livers in FRG mice.

Incorporation of human fetal liver mesenchymal cells into hEHOs to develop a co-culture hepatic organoid

Previously, a 3D co-culture in vitro liver system was established for toxicity assessment and fibrosis, which was generated from the combination of human hepatocyte line HepaRG and hepatic stellate cells, one of the types of mesenchymal cells in liver. ${ }^{32,33}$ Based on the findings of previous reports, a similar strategy was designed to utilize hEHOs for establishing the improved models of human liver diseases, such as Alcoholic Liver disease (ALD). Human fetal liver mesenchymal cells (hFLMCs) were first chosen as components in helping hEHOs because they are at primitive lineage stage similar to $\mathrm{hEHOs}$ and may have potential to beneficially support hEHOs' differentiation to hepatocytes as a micro-environment. ${ }^{34,35}$ The hFLMCs in culture mainly consisted of PDGFR- $\beta^{+}$cells $(99 \%)$ or LRAT $^{+}$cells $(97 \%)$ (Supplementary information, Fig. S9a); both of the PDGFR- $\beta$ and LRAT were the well-defined markers for hepatic stellate cells (HSCs). ${ }^{3,36,37}$ A protocol suitable for the co-culturing of hFLMCs with hEHOs to form co-culture hepatic organoid was then established (See the Methods for detailed information). After the two types of cells were combined at a ratio of 2:1 (hEHO: hFLMC) and seeded into the established 3D co-culture system, the cells self-organized into mixed 3D clusters by day 2 after seeding. Then, the 3D cluster of mixed cells became a typical form of organoid of about $150-200 \mu \mathrm{m}$ in diameter by day 14 (Supplementary information, Fig. S9b), referred to as hFLMC/hEHO. The cells positive for the mature hepatocyte marker ALB were found located peripherally in the organoids, while the PDGFR- $\beta^{+}$hFLMCs were located primarily in the centers of the organoids (Fig. 7a). Remarkably, ethanol (EtOH) metabolism-associated enzymes, both alcohol dehydrogenase $(\mathrm{ADH})$, and CYP2E1 were found in hFLMC/hEHO by immunostainings (Fig. 7a), suggesting that the hFLMC/hEHO should perform the relative functions of EtOH metabolism and should be usable as a practicable model system for detecting the effects of EtOH on liver. Furthermore, the co-culture with hFLMCs promoted the hepatic differentiation of hEHOs, which was shown from the results of the increase in levels of hepatic functions, including albumin secretion, urea production and CYP3A4 activity (Fig. 7b).

$\mathrm{hFLMC/hEHO}$ enabled modeling of alcoholic liver injury After confirming the expression of key enzymes for EtOH metabolism, pathophysiological parameters were evaluated in $\mathrm{hFLMC} / \mathrm{hEHO}$ with the $\mathrm{EtOH}$ treatment for 7 days. During the process, increase of CYP2E1 activity was observed consistent with its inducible feature (Fig. 7c). The hFLMC/hEHO displayed liver damage as indicated by up-regulated secretion of ALT, AST and lactate dehydrogenase (LDH) and also displayed a reduction in cell viability shown by increased Dead/Live content ratio (Fig. 7d, e, Supplementary information, Fig. S9c). Furthermore, the hFLMC/ $\mathrm{hEHO}$ showed the fibrogenic responses to EtOH as evidenced by up-regulated gene expression of fibrogenic markers (LOXL2, COL1A1, COL3A1, ACTA2 and TGF 1 1) (Fig. 7f). They were positive for collagen type 1(Col1), pro-a1 collagen type I (Col1a1), Desmin, a-SMA, Sirius red and pro-collagen type I secretion (Fig. 7g), further confirming the generation of fibrosis and increased deposition of extracellular matrix after $\mathrm{EtOH}$ treatment. These metabolic changes in hFLMC/hEHO recapitulated ALD-associated pathophysiology.

Next, we tested whether EtOH-treated hFLMC/hEHO could recapitulate the currently known mechanism of ALD. It has been indicated that $\mathrm{ADH}$ and CYP2E1 metabolized EtOH to the toxic acetaldehyde accompanied by the production of reactive oxygen species (ROS) that further induce oxidative stress and steatosis in ALD. ${ }^{38}$ To test if these ALD-associated molecular changes were detectable in our model, levels of both oxidative stress and steatosis were measured in hFLMC/hEHO, hEHO-Heps and hFLMCs. Based on our previously established method for detecting cytotoxicity of hepatotoxicants, ${ }^{39}$ CellROX ${ }^{\mathrm{TM}}$ Deep Red Reagent (CellROX) and Monochlorobimane $(\mathrm{mBCl})$ were used to measure the reactive oxygen species (ROS) and glutathione (GSH) respectively in cells, which represented the status of oxidative stress. EtOH treatment led to enhanced oxidative stress that was indicated by the increase of CellROX and reduction of $\mathrm{mBCl}$ in $\mathrm{hFLMC/hEHO}$ and hEHO-Heps, but not in hFLMCs. In the meanwhile, hFLMC/hEHO displayed the most significant effect of EtOH treatment (Fig. 8a, Supplementary information, Fig. S9d). Because acetaldehyde can lead to structural and functional alterations of mitochondria, ${ }^{38,40}$ MitoProbe ${ }^{\mathrm{TM}} \mathrm{JC}-1$ (JC-1) was applied to quantify alternations in mitochondrial membrane potential (MMP) of the three cell-types. The decrease of the ratio of JC- 1 red to green was found after EtOH treatment, confirming the mitochondrial membrane potential depolarization, which was also most obvious in $\mathrm{hFLMC/hEHO}$ but not in hFLMCs (Fig. 8b, Supplementary information, Fig. S9e). Oxidative stress and mitochondrial disorder were previously reported to promote steatosis by disrupting hepatic lipid metabolism in ALD. ${ }^{38}$ Next, whether the steatosis existed in our model following $\mathrm{EtOH}$ treatment was determined. Through localization of neutral lipid droplets within cells, excessive accumulation of fat was found in hFLMC/hEHO, hEHOHeps but not in hFLMCs (Fig. 8c, Supplementary information, Fig. S9f). Genes encoding the lipogenic-associated enzymes ( $A C C 1$, FASN and $S C D$ ) and transcription factors (SREBP1 and PPAR- $\gamma$ ) were found up-regulated by EtOH treatment (Fig. 8d). Remarkably, it was found that the expression of ACC1 (rate-limiting enzyme of lipogenesis) was increased at both transcriptional level and translational level after EtOH treatment, which further suggested 


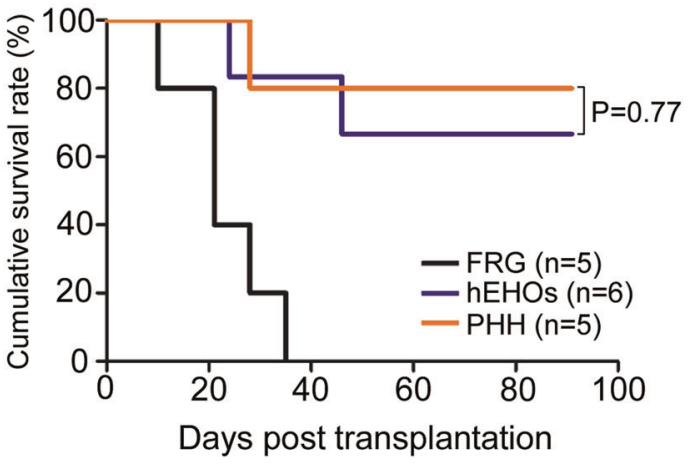

C

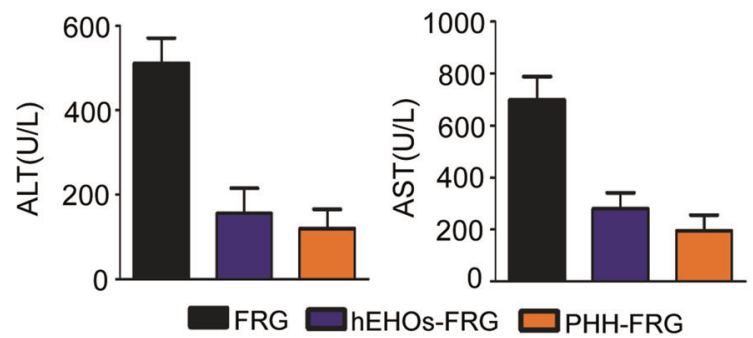

e

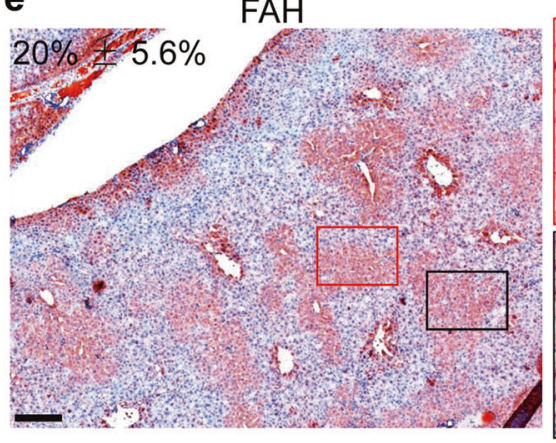

g

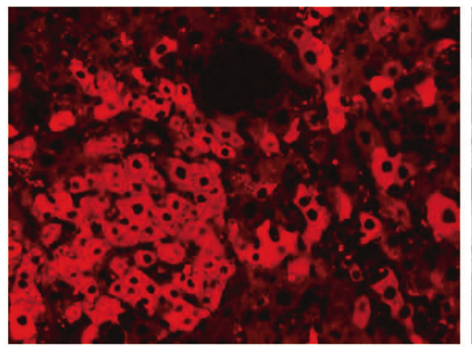

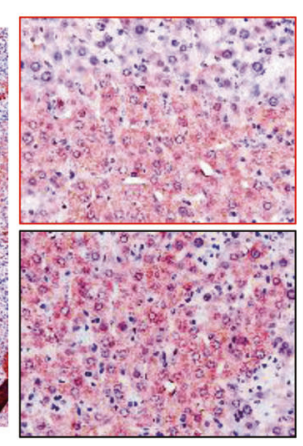

$\mathrm{m}$ and hCK18

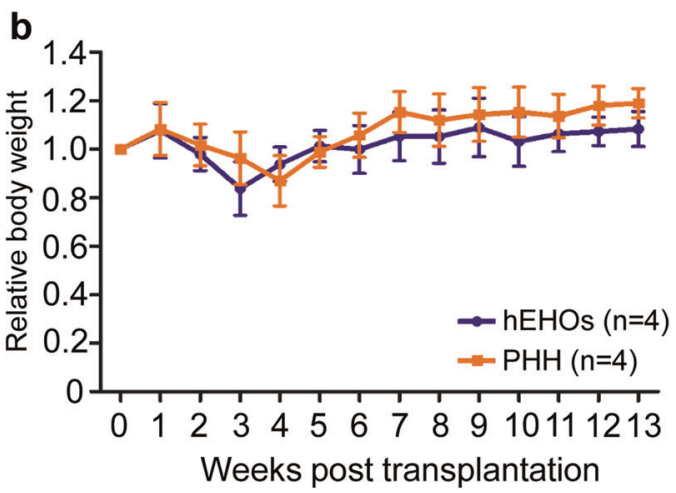

d

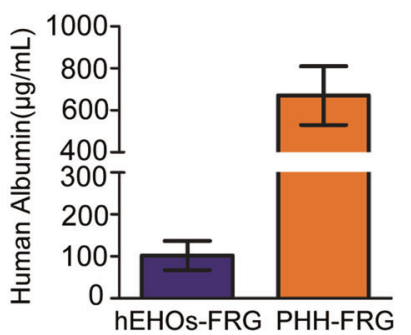

f hALB

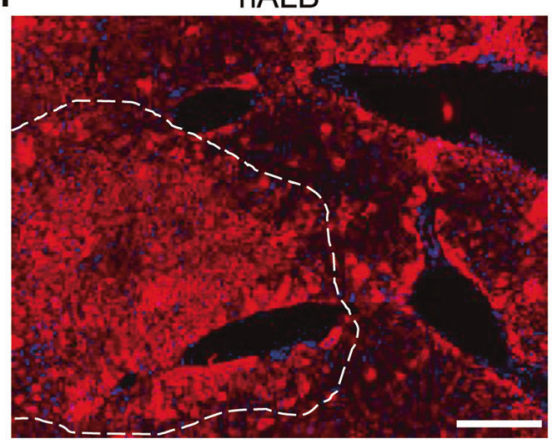

h $\quad$ hALB

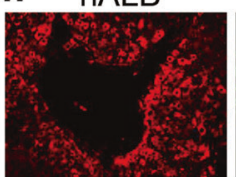

$m$ and $h G S$

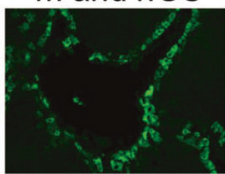

Merge

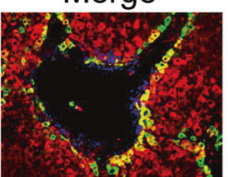

hALB

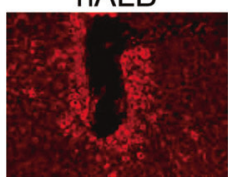

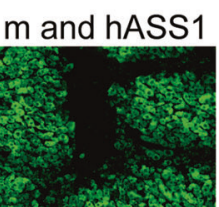

Merge

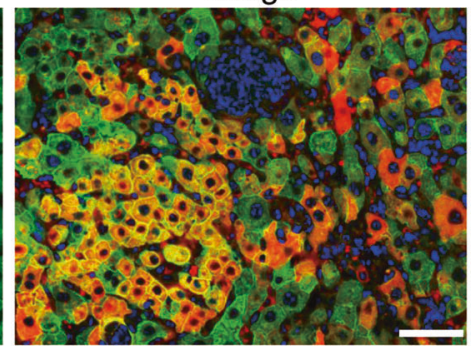

Merge

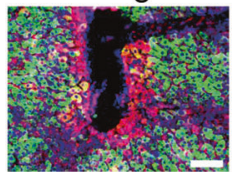

Fig. 6 The hEHOs highly repopulate the liver parenchyma of FRG mice. a Kaplan-Meier survival curve of FRG mice with or without transplantation of hEHOs (hEHOs-FRG), or PHH (PHH-FRG). b The body weight changes of hEHOs-FRG and PHH-FRG mice. c ALT and AST levels in moribund control FRG mice $(n=3)$, surviving hEHOs-FRG mice $(n=4)$, and surviving PHH-FRG mice $(n=4)$ at 3 months after transplantation. d Human serum albumin levels in recipients of hEHOs $(n=4)$ or $\mathrm{PHH}(n=4)$ were measured at 3 months after transplantation. Immunostaining using anti-FAH antibody (e) and anti-human-specific ALB (hALB) antibody (f) in hEHOs-FRG mice at 3 months after transplantation. g Co-immunostainings for hALB and human and mouse CK18 (m and hCK18). $h$ Co-immunostainings for hALB and liver zonation markers, the GS ( $\mathrm{m}$ and hGS), and the ASS1 (m and hASS1). Scale bar: $700 \mu \mathrm{m}(\mathbf{e}), 200 \mu \mathrm{m}(\mathbf{f}), 50 \mu \mathrm{m}(\mathbf{g}), 100 \mu \mathrm{m}(\mathbf{h})$ 
a

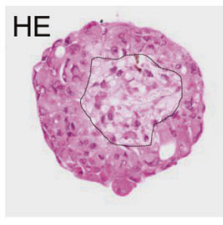

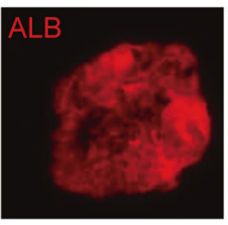
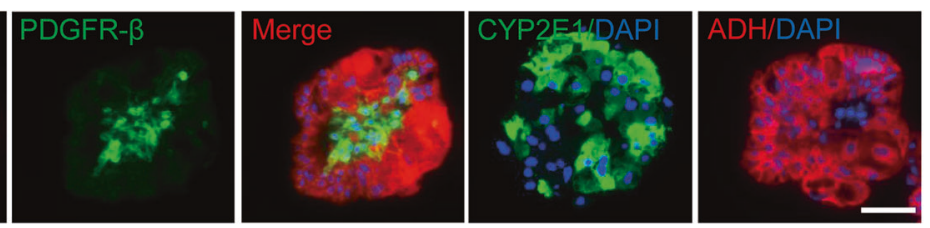

b
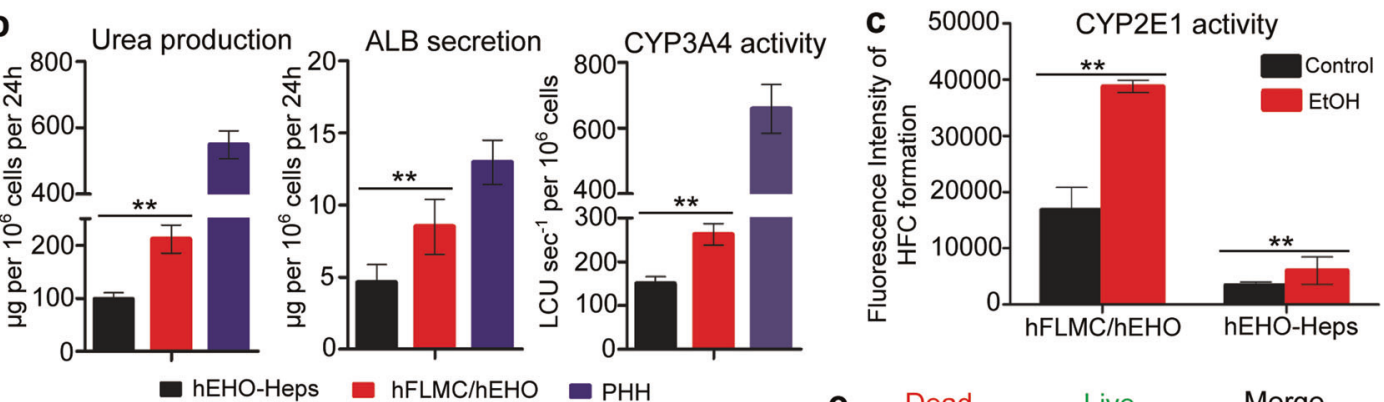

d

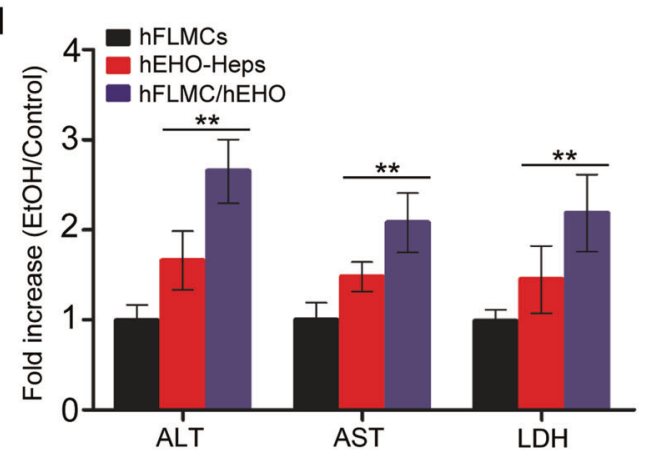

f
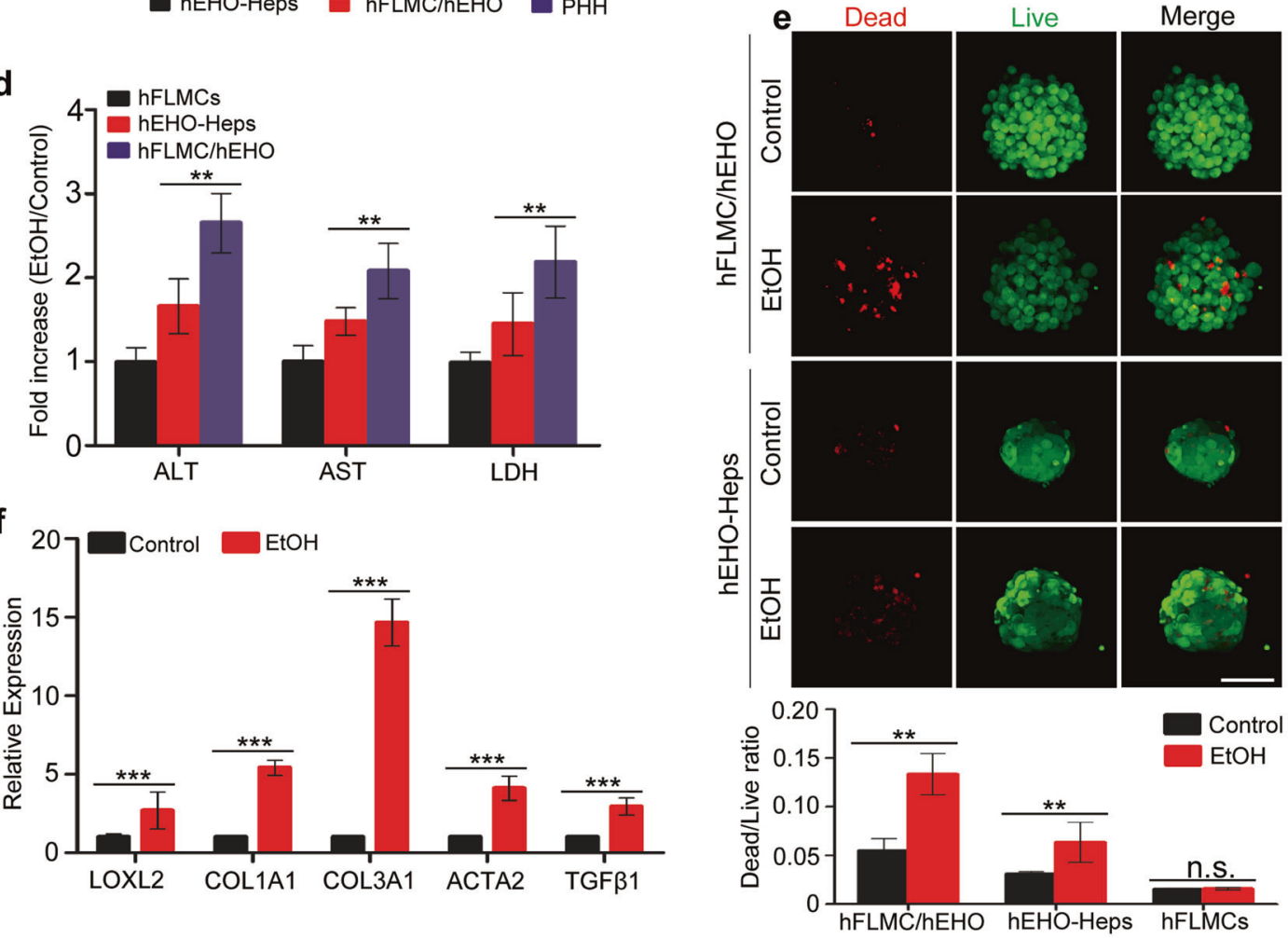

g
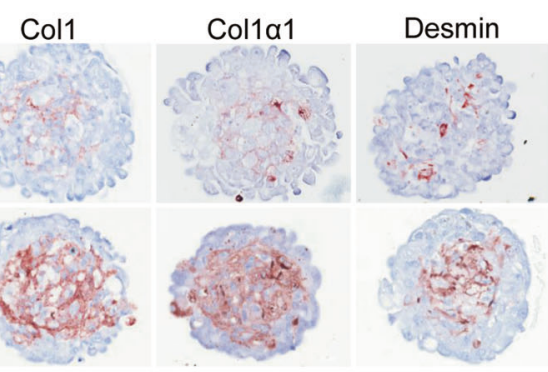

a-SMA
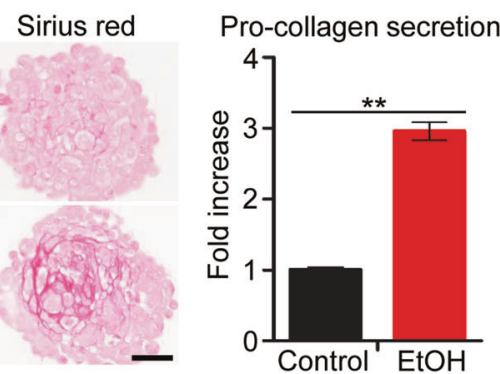

increasing fatty acid biosynthesis by $\mathrm{EtOH}$-induced oxidative stress $^{41}$ (Fig. 8e).

In addition, inflammation also has been reported to play important roles in the pathogenesis of ALD. ${ }^{42}$ Therefore, the release of inflammatory mediators was evaluated in the $\mathrm{EtOH}$ - treated $\mathrm{hFLMC/hEHO}$. Results of secretome analyses by cytokine arrays revealed that both cytokines relative to chemokines (I-309, MIP-1d, MCP-2, MIP-1a and EOTAXIN) and interleukins (IL-3, IL-12 p40, IL-1 $\beta, I L-17, I L-10$ and IL-6) increased in the experimental group of EtOH-treated hFLMC/hEHO when compared with the 
Fig. 7 Generation of hFLMC/hEHO organoid to model ethanol-induced liver injury. a Representative HE staining of paraffin sections of $\mathrm{hFLMC/hEHO}$ and immunostaining of hepatic marker (ALB), mesenchymal cell marker (PDGFR- $\beta$ ), and the major ethanol-oxidizing enzymes (ADH and CYP2E1) in hFLMC/hEHO. b Representative assays of hepatocyte cellular functions in hEHO-Heps and hFLMC/hEHO. c Assessment of CYP2E1 activity of hEHO-Heps, and hFLMC/hEHO in the presence or the absence of EtOH for 7 days using fluorescence-based assays. d Analysis for ALT, AST and LDH levels in hFLMCs, hEHO-Heps and hFLMC/hEHO supernatants after 7 days treatment of $100 \mathrm{mM}$ EtOH. e Assessment of cell viability in hEHO-Heps and hFLMC/hEHO by Dead/Live staining at day 7 of EtOH treatment. $\mathrm{fRT}$-PCR of fibrogenic genes in $\mathrm{hFLMC/hEHO}$ after EtOH treatment for 7 days. Results are presented as mean \pm SD from 3 replicates from three independent repeated experiments. g Representative pictures of Col1, Col1 $\alpha 1$, desmin, a-SMA, and Sirius red stainings of paraffin sections of hFLMC/hEHO and assay for pro-collagen type I secretion after exposure to EtOH for 7 days. Data are presented as mean \pm SD $(n=3$ per group in $\mathbf{c}$, $\mathbf{d}, \mathbf{g}$, and $n=6$ per group in e). ${ }^{* *} P<0.01 ;{ }^{* *} P<0.001$. Scale bar: $50 \mu \mathrm{m}(\mathbf{a}, \mathbf{g}), 100 \mu \mathrm{m}(\mathbf{e})$

group of EtOH-untreated hFLMC/hEHO as a control (Fig. 8f, Supplementary information, Table S3). Furthermore, Gene Ontology (GO) analyses on these members of up-regulated mediators revealed that interleukin-1 signaling was highly activated (Supplementary information, Fig. S9g), which was consistent with the previous findings that IL-1 $\beta$, an important pro-inflammatory cytokine, was up-regulated in patients of alcoholic hepatitis. ${ }^{43,44}$ Besides interleukin-1 signaling, IL-17, a potent pro-inflammatory cytokine participating in ALD by promoting both liver inflammation and fibrosis, and a characteristic symbol of alcoholic patients with liver disease, ${ }^{45}$ was also found increased in EtOH-treated hFLMC/hEHO (Fig. 8f, Supplementary information, Fig. S9h).

In summary, all of above results indicate that the hFLMC/hEHO were modified hepatic organoids with improved properties enabling these organoids to be a reliable and practical model for studying details of activities in ALD and also for exploring the underlining mechanisms as well (Supplementary information, Fig. S10).

\section{DISCUSSION}

Hepatocytes are the primary entities in various liver diseases and also critical sites for xenobiotic metabolism of drugs and toxins as well as their toxic derivatives. Currently, large numbers of human hepatocytes with fully sustained differentiated functions are required for high-throughput drug screenings, disease modeling and cell-based therapy applications. An ideal strategy is to use in vitro expansion of hepatic stem/progenitor cells and then subject them to differentiation conditions to obtain large quantities of mature hepatic cell derivatives. With respect to this need, we pursued establishment of a scalable and defined protocol involving two distinct and interdependent steps. The first step was to establish a 3D expansion system for the replication of $\mathrm{hESC}$-derived, bipotential hepatic organoids that could provide sufficient numbers of hepatic cells. The second step was to promote the hepatic maturation of hEHOs. Here, our 3D culture conditions for both maintenance and differentiation of hEHOs were successful using chemically defined, feeder-/serumfree media. Cells of hEHOs showed the phenotypic traits of hepatic lineage restriction with potential of differentiation into functional hepatocytes and cholangiocytes. Remarkably, hEHOs were successfully expanded on an industrial scale in vitro and demonstrated no evidence of tumorigenicity after transplantation in vivo. We believe that the combination of those two steps will supply functional hepatocytes in sufficient quantity and safety. Therefore, cells of hEHOs displayed ideal properties of hepatic stem/progenitor cells and can be used as cell sources for potential applications in cell therapies, disease modeling, and models for the pharmaceutical industry.

Although the refined culture platform in previously reported studies could massively produce the hPSC-derived liver buds to a relevant large scale $\left(>10^{8}\right)$, it was still difficult to generate the cells to the levels up to $10^{10}$-scale usually required for the potential therapeutic applications in clinical transplantation needed for human adults. ${ }^{46}$ The hAHOs that were derived from adult bile duct contained bipotential liver stem cells showing the capacity of long-term propagation in vitro. ${ }^{9}$ However, they had limited potential of differentiation into mature hepatocytes in vitro and in vivo. The adult $\mathrm{PHH}$-derived organoids demonstrated significant engraftment and cell expansion after transplantation into the injured livers of murine recipients. ${ }^{10}$ However, the in vitro expansion of the cells inside these organoids was with a limited expansion time of less than 2.5 months. Remarkably, our hEHOs had the advantage of the capacity of extensive in vitro cell expansion, which could reach to the level of up to $10^{18}$ cells after 5 months under our specially established in vitro culture condition. Besides, our hEHOs displayed considerable hepatic bipotentiality in vitro and also had the capacity of significant engraftment into damaged liver parenchyma in vivo.

Moreover, another remarkable improvement was that hFLMC/ $\mathrm{hEHO}$ was generated by the first applicable strategy to successfully mimic the pathophysiological changes and pathogenesis of ALD. ALD is the primary cause of liver-related mortality in Western countries. Currently, there are still no FDA-approved drugs for treating ALD, partially due to the lack of a suitable model that can reliably mimic the human pathophysiology and can be used to identify an effective drug for ALD treatments. ${ }^{38}$ Therefore, the $\mathrm{hFLMC/hEHO}$ should provide a revolutionary tool to study the mechanisms underlying diseases and enable industrial-scaled screening of new drugs to prevent or cure ALD. Next, immune cells such as Kupffer cells will be introduced into hFLMC/hEHO enabling analysis of steatohepatitis, which may provide in the future a more accurate in vitro model for biomedical research of ALD. In summary, our protocols for generating hFLMC/hEHO offer a reliable and practical ex vivo system for modeling the pathophysiology of ALD for the first time.

\section{MATERIALS AND METHODS}

Human liver tissues

Human adult liver tissues were obtained from donors during liver transplantation performed at the Beijing Friendship Hospital with patients' informed consent. Human fetal liver tissues at 26 gestational weeks were obtained from aborted tissues with patients' informed consent. These experiments were approved by the Academic Committee of Beijing Institute of Health Service and Transfusion Medicine and the Ethics Committees of Beijing Friendship Hospital.

Animals

All mice experiments used the ARRIVE guidelines and were approved by the Institutional Animal Care and Use Committee at Beijing Institute of Health Service and Transfusion Medicine.

Generation and expansion of hEHOs from human embryonic stem cells

The schematic of the step-wised differentiation procedures has been shown in Fig.1a. The H9 Human ESC line was purchased from WiCell Research Institute (Madison, Wisconsin, USA). H9 was maintained on feeder-free conditions in TeSR-E8 media (Stem Cell) in six-well plates (Corning) coated with growth-factor-reduced Matrigel (Corning). To induce DE differentiation, cells were cultured in RPMI1640 
a

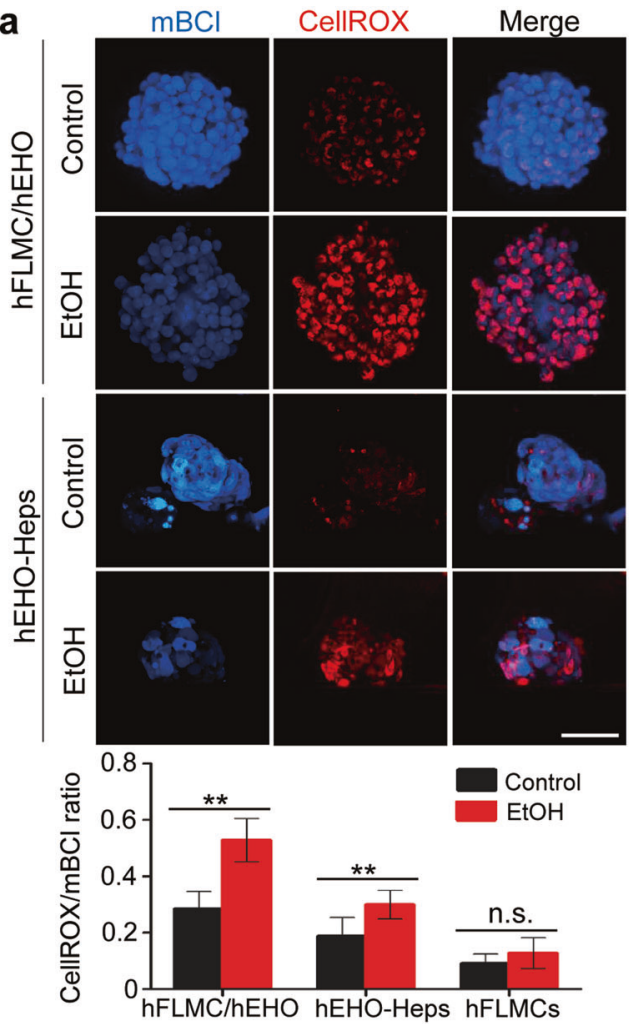

C
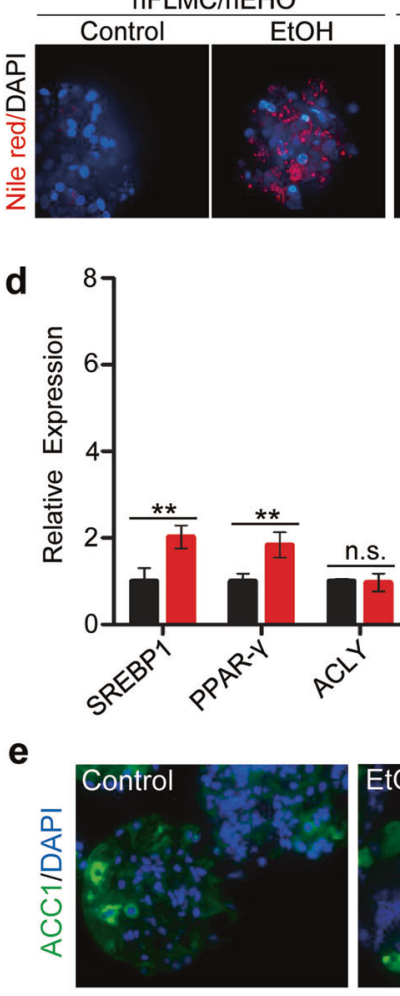

b
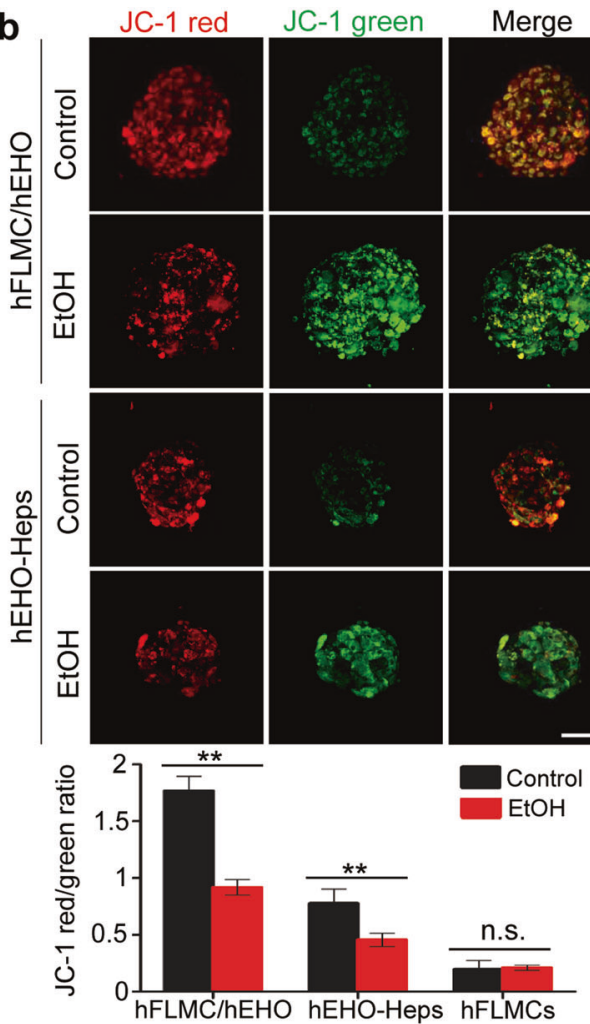

产
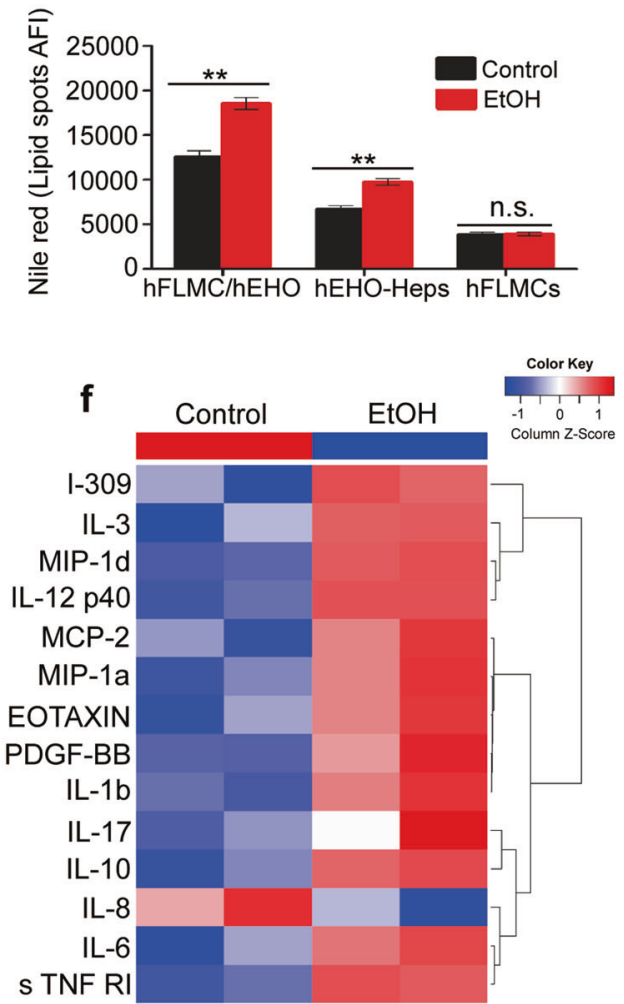

medium (Gibco) supplemented with 1\% B27 (Gibco), $100 \mathrm{ng} / \mathrm{ml}$ Activin A, $50 \mathrm{ng} / \mathrm{ml}$ Wnt3A (both from R\&D) for 1 day and transferred to RPMI1640 supplemented with $2 \%$ B27, $100 \mathrm{ng} / \mathrm{ml}$ Activin A for the next 2 days. To induce cells to reach HS stage, DE cells were cultured in hepatocyte culture medium (HCM, Lonza, CC-3198) without EGF, supplemented with $10 \mathrm{ng} / \mathrm{ml}$ FGF-2, $20 \mathrm{ng} / \mathrm{ml}$ BMP4 (both from R\&D) in the subsequent 5 days. Then the cells were dissociated into single cells with $0.25 \%$ Trypsin-EDTA (Gibco) and embedded in a 30-50 $\mu \mathrm{L}$ droplet of Matrigel or BME 2 (R\&D), at a density of 3000-10,000 cells per well in ultra-low attachment surface 
Fig. $8 \mathrm{hFLMC/hEHO}$ organoid enables recapitulation of ALD-associated pathogenesis. a Parameters of oxidative stress are assessed by molecular probes CellROX, and $\mathrm{mBCl}$ in hEHO-Heps and $\mathrm{hFLMC/hEHO}$ at day 7 after EtOH treatment. b Assessment of mitochondrial membrane potential by molecular probes JC-1 in hEHO-Heps and hFLMC/hEHO at day 7 after EtOH treatment. c Assessment of steatosis by Nile Red staining in hEHO-Heps and hFLMC/hEHO at day 7 after EtOH treatment. d qRT-PCR of Lipogenic-associated genes in hFLMC/hEHO at day 7 after $\mathrm{EtOH}$ treatment. Results are presented as mean $\pm \mathrm{SD}$ from 3 replicates from three independent repeated experiments. e Immunostaining for ACC1 in hFLMC/hEHO at day 7 after EtOH treatment. $f$ Secretome analysis of inflammatory mediators in the supernatant of EtOH-treated $\mathrm{hFLMC/hEHO}$ at day 7 versus control group. A list of secreted proteins with $>1.2$-fold or $<0.83$-fold changes (absolute logFC $>$ $0.263)$ is shown. Data are presented as mean \pm SD $\left(n=6\right.$ per group in a-c). ${ }^{* *} P<0.01 ;{ }^{* *} P<0.001 ;$ n.s. $=$ not significant $(P>0.05)$. Scale bar: $50 \mu \mathrm{m}(\mathbf{e}), 100 \mu \mathrm{m}(\mathbf{a}-\mathbf{c})$

24-well plates (Corning). After incubating the plates at $37^{\circ} \mathrm{C}$ for $7-8$ min for gelation, culture medium consisting of Advanced DMEM/ F12 supplemented with $1 \%$ Glutamax, 10 mM HEPES, 1\% N2, 1\% B27 (all from Gibco), $1.25 \mathrm{mM} \mathrm{N}$-Acetylcysteine, $10 \mathrm{nM}$ gastrin, $10 \mathrm{mM}$ Nicotinamide (all from Sigma), $5 \mu \mathrm{M}$ A83-01, $10 \mu \mathrm{M}$ Forskolin (both from Selleck), $250 \mathrm{ng} / \mathrm{ml}$ R-spondin1, $100 \mathrm{ng} / \mathrm{ml} \mathrm{Wnt3a}$ and $50 \mathrm{ng} / \mathrm{ml}$ EGF (all from R\&D) was added, with medium changed every 2 days for 7-10 days. For organoids passaging, hEHOs were dissociated into small fragments with Accutase (Sigma) and split at 1:6-8 every 7-9 days. Those small fragments could be cryopreserved in serum-free cryopreservation medium (Stem Cell). The frozen pieces could be thawed following standard procedures and cultured in the above medium if needed. For growth curve of hEHOs, the organoids grown in expansion medium were dissociated into single cells with Accutase. Based on the basic formula of the expantion curve $y(t)=y_{0} \times e^{(\text {growth rate } \times t)}(y=$ cell numbers at final time point; $\mathrm{y}_{0}=$ cell numbers at initial time point; $\mathrm{t}=$ time) and previous reports about mouse pancreas organoids ${ }^{6}$ and human primary hepatic organoids growth curves, ${ }^{9}$ we derived the growth rate of hEHOs. The doubling time was calculated as doubling time $=\ln (2) /$ growth rate for each time window analyzed.

\section{Differentiation of hEHOs into mature hepatocytes (hEHO-Heps) in vitro}

In order to generate mature hepatocytes, the hollow hEHOs in the gel were pretreated with Advanced DMEM/F12 supplemented with $0.1 \%$ BSA, $1 \%$ Glutamax, $10 \mathrm{mM}$ HEPES, 1\% N2, 1\% B27, $1.25 \mathrm{mM}$ N-Acetylcysteine, $10 \mathrm{mM}$ Nicotinamide, $10 \mathrm{nM}$ gastrin, $25 \mathrm{ng} / \mathrm{ml} \mathrm{BMP7}, 25 \mathrm{ng} / \mathrm{ml} \mathrm{FGF} 4$ and $50 \mathrm{ng} / \mathrm{ml} \mathrm{HGF}$ (growth factors all from R\&D) for 4 days. ${ }^{9}$ Then the pretreated-hEHOs were isolated from the gel and dissociated to single cell with Accutase and seeded in ultra-low attachment surface 24-well plates at a density of $1-1.5 \times 10^{5}$ cells per well to generate threedimensional aggregates. Aggregates were cultured in $\mathrm{HCM}$ without EGF or Hepatocyte Medium (HM, Sciencell), supplemented with $0.1 \% \mathrm{BSA}, 1 \% \mathrm{~B} 27,0.2 \mathrm{mM}$ L-ascorbic acid trisodium salt (Sigma), $1.25 \mathrm{mM} \mathrm{N}$-Acetylcysteine, $10 \mu \mathrm{M} \mathrm{Y-27632} \mathrm{(Stemcell),}$ $20 \mathrm{ng} / \mathrm{ml} \mathrm{HGF}, 20 \mathrm{ng} / \mathrm{ml}$ OSM (both from R\&D), $0.5 \mu \mathrm{M}$ dexamethasone (Dex, Sigma) for 3 days. After this stage, the medium was changed to HCM or HM, supplemented with $0.1 \%$ BSA, $1 \%$ B27, 0.2mM L-ascorbic acid trisodium salt, $1.25 \mathrm{mM} \mathrm{N}$-Acetylcysteine, $10 \mu \mathrm{M}$ Y-27632, 1\% ITS (Gibco), $0.5 \mu \mathrm{M}$ Dex, $10 \mathrm{ng} / \mathrm{ml}$ EGF, $100 \mu \mathrm{M}$ 8-Br-cAMP (Selleck), $10 \mu \mathrm{M}$ VK2, $10 \mu \mathrm{M}$ LCA (both from Sigma) with medium changes every 2 days for 8 days. ${ }^{11,47}$ This differentiation protocol was applied for the assays of hEHO-Heps in Figs. 4, 5 and Supplementary information, Fig. S5.

Co-culture of hFLMCs with hEHOs (hFLMC/hEHO organoid) and modeling of ALD

hFLMCs were isolated as described below. The protocol for generation of $\mathrm{hFLMC/hEHO}$ organoid was different from the protocol for generation of hEHO-Heps. Specifically, in order to form $\mathrm{hFLMC/hEHO}$ co-culture organoid inside of wells of 96 -well plates, sterile $0.8 \%$ agarose was used for pre-coating. The hEHOs and hFLMCs were dissociated into single cells and re-suspended at a ratio of $2: 1$ in Advanced DMEM/F12 plus $0.1 \%$ BSA and $1 \%$ B27, supplemented with $0.2 \mathrm{mM}$ L-ascorbic acid trisodium salt, $1 \%$
Glutamax, 1.25mM N-Acetylcysteine, $10 \mu \mathrm{M} \mathrm{Y-27632}$ and $20 \mathrm{ng} / \mathrm{ml}$ HGF, with medium changes every 2 days for 7 days. After 7 days of initiation, the medium was changed to HCM without EGF, supplemented with $20 \mathrm{ng} / \mathrm{ml}$ OSM and $0.5 \mu \mathrm{M}$ Dex for another 7 day, with medium refreshed every 2 days. In order to make in vitro ALD model using hFLMC/hEHO, $100 \mathrm{mM}$ ethanol (EtOH) was added into the culture medium for 7 days with medium refreshed every day. ${ }^{48,49}$ Parafilm was used to seal the 96-well plate to limit EtOH evaporation. The control group without EtOH was treated in the same way. This differentiation protocol was applied for the assays of hFLMC/hEHO and their-associated ALD model in Fig. 7, Fig. 8 and Supplementary information, Fig. S9.

Spontaneous differentiation of hEHOs in vivo

$2-3.0 \times 10^{5}$ cells dissociated from hEHOs were mixed with 100-200 $\mu \mathrm{L}$ Matrigel and were wrapped in each of the epididymal fat pads of male 8-10-week-old NOD/SCID mice $(n=6$, Beijing Vital River Laboratory Animal Technology Company). After 4-10 weeks, the mixed transplants were surgically removed, fixed and embedded in paraffin, and analyzed using H\&E staining and immunostaining.

Transplantation of cells into FRG mice

For in vivo engraftment of hEHOs and $\mathrm{PHH}$ into the liver parenchyma, Male $\mathrm{Fah}^{-/-} / \mathrm{Rag}^{-/-} / \| 2 \mathrm{rg}^{-/-}$(FRG) mice (6-8 weeks old, Beijing IDMO Co., Ltd.) were maintained with NTBC in the drinking water at $7.5 \mathrm{mg} / \mathrm{L}$. $1 \times 10^{6}$ cells from hEHOs or PHHs were suspended in $30 \mu \mathrm{L}$ of Matrigel and injected directly into left lower lobes of the livers of FRG mice (with hEHOs $n=6$, with PHH $n=5$ ). NTBC was withdrawn from the drinking water following the stepwise schedule of concentrations at $100 \%, 25 \%, 12 \%, 6$ and $0 \%$ after transplantation. NTBC at concentration of $50 \%$ was transiently given again for three days when mice lost nearly $20 \%$ of their starting body weights. FRG mice transplanted with the cells were sacrificed for analysis at 3 months after transplantation. Human albumin in mice serum was detected and quantified using the Human Albumin ELISA kit (Bethyl Laboratory).

Isolation of hFLMCs

Fetal liver tissue was cut into $1-3 \mathrm{~mm}^{3}$ fragments for digestion in DMEM (Gibco) containing collagenase type IV at $300 \mathrm{U} / \mathrm{ml}$ and DNase I at $30 \mathrm{mg} / \mathrm{ml}$ (both from Sigma) at $37^{\circ} \mathrm{C}$ for $30-60 \mathrm{~min}$. The digestion suspensions were then filtered through a $70 \mu \mathrm{m}$ Nylon cell strainer and centrifuged for $5 \mathrm{~min}$ at $50 \mathrm{~g}$ at $4{ }^{\circ} \mathrm{C}$. The supernatant was transferred to new tubes, and centrifuged at $1100 \mathrm{rpm}$ for $5 \mathrm{~min}$ at $4{ }^{\circ} \mathrm{C}$. The cell pellets were washed with DMEM twice and then cultured in Williams' $\mathrm{E}$ medium supplemented with 5\% FBS, 1\% Glutamax (all from Gibco).

\section{Generation of FSCs and $\mathrm{HB}$}

To generate FSCs, hESC-derived DE cells were cultured in RPMI 1640 medium supplemented with $2 \%$ B27 and $50 \mathrm{ng} / \mathrm{ml}$ Activin A for 3-4 days. Then the cells were digested and re-seeded on Matrigel-coated plates in RPMI 1640 medium containing B27 Supplement, NEAA, $10 \mathrm{ng} / \mathrm{ml}$ Activin A, $20 \mathrm{ng} / \mathrm{ml} \mathrm{bFGF,} 10 \mathrm{ng} / \mathrm{ml}$ BMP4, $20 \mathrm{ng} / \mathrm{ml} \mathrm{HGF}$, and $50 \mathrm{ng} / \mathrm{ml} \mathrm{EGF}{ }^{23}$ To generate $\mathrm{HB}$, the cells of HS stage were cultured in HM medium with $1 \%$ B27, 
$0.2 \mathrm{mM}$ L-ascorbic acid trisodium salt, $4.5 \times 10^{-4} \mathrm{M}$ MTG (Sigma), $20 \mathrm{ng} / \mathrm{ml} \mathrm{HGF}, 0.5 \mu \mathrm{M}$ Dex, and $20 \mathrm{ng} / \mathrm{ml}$ OSM for 9 days. ${ }^{24}$

$\mathrm{PHH}$ isolation and culture

Hepatocytes were isolated using a modified two-step collagenase perfusion technique. ${ }^{50}$ In brief, the liver tissue was flushed with ice-cold HBSS medium with $0.5 \mathrm{mM}$ EGTA and $0.5 \%$ BSA for 15-30 $\mathrm{min}$ and followed with perfusion with $1 \mathrm{mg} / \mathrm{ml}$ collagenase type IV for $20-40 \mathrm{~min}$ at $37^{\circ} \mathrm{C}$. The resulting cell suspensions were filtered through a $100 \mu \mathrm{m}$ Nylon mesh, and centrifuged for $3 \mathrm{~min}$ at $75 \mathrm{~g}$. The cell pellets were re-suspended in medium with $35 \mathrm{ml}$ DMEM/F12,13.5 ml Percoll (GE healthcare, density $1.130 \mathrm{~g} / \mathrm{ml}$ ) and $1.5 \mathrm{ml} 10 \times$ HBSS and were centrifuged at $100-150 \mathrm{~g}$ for $10 \mathrm{~min}$ at room temperature. The cell pellets were washed 3 times in DMEM/F12. The primary human hepatocytes were then resuspended in Williams' $\mathrm{E}$ medium supplemented with $5 \%$ FBS and seeded in a collagen I coated dish. After $4-10 \mathrm{~h}$ of cell attachment, the culture medium was changed to HM medium with daily medium changes. Primary human hepatocytes were cultured in HM medium for 2 days before functional assays.

\section{hAHOs culture}

The hAHOs were generated and cultured using previously described methods. ${ }^{9,51}$ Briefly, the isolated EpCAM $^{+}$cells were mixed with Matrigel or BME2, and $3000-10,000$ cells were seeded per well in ultra-low attachment surface 24 -well plates. The culture medium was consisted of Advanced DMEM/F12 supplemented with $1 \% \mathrm{~N} 2$ and $1 \% \mathrm{~B} 27,1.25 \mathrm{mM} \mathrm{N}$-Acetylcysteine, $10 \mathrm{nM}$ gastrin, $10 \mathrm{mM}$ Nicotinamide, $1 \%$ Glutamax, $5 \mu \mathrm{M}$ A83-01, $10 \mu \mathrm{M}$ Forskolin, $250 \mathrm{ng} / \mathrm{ml}$ R-spondin 1, $50 \mathrm{ng} / \mathrm{ml} \mathrm{EGF,} 100 \mathrm{ng} / \mathrm{ml} \mathrm{FGF10,}$ and $25 \mathrm{ng} / \mathrm{ml} \mathrm{HGF}$. For the first 2 days, $10 \mu \mathrm{M}$ Y-27632 was added to the culture medium. After 10-14 days, organoids were passaged as previously described.

\section{Karyotype analysis}

Exponentially growing hEHOs at passage 1 and 15 were incubated with $40 \mathrm{ng} / \mathrm{ml}$ colchicine (Sigma) for $4 \mathrm{~h}$. Then $\mathrm{hEHOs}$ were dissociated into single cells with Accutase and processed using a standard karyotyping protocol.

Flow cytometry

Single cell suspensions were obtained by dissociation with Accutase for $3-5 \mathrm{~min}$ at $37^{\circ} \mathrm{C}$. For cell surface antigen analyses, staining was performed in PBS at $4{ }^{\circ} \mathrm{C}$ for $30 \mathrm{~min}$. For intercellular protein analyses, staining was performed on 4\% PFA-fixed cells. After blocking with $10 \%$ Goat or Donkey serum and incubation with primary antibodies for $1 \mathrm{~h}$, the cells were incubated with secondary antibodies for $40 \mathrm{~min}$ in the dark, and finally passed through $70 \mu \mathrm{m}$ cell strainers. The stained cells were analyzed or sorted with BD FACSCalibur (BD Biosciences), and the data were analyzed using the FlowJo software (TreeStar). The sources and concentrations of primary, secondary antibodies and isotype controls are listed in Supplementary information Table S1.

Differentiation of hEHOs into cholangiocytes in vitro

The hEHOs were dissociated into single cells and re-suspended in $1.2 \mathrm{mg} / \mathrm{mL}$ ice-cold collagen type I (Corning) at a concentration of $1 \times 10^{5} / \mathrm{ml}$ cells in 24-well plates. The cholangiocytes differentiation medium was based on Williams' E supplemented with $10 \mathrm{mM}$ Nicotinamide, $0.2 \mathrm{mM}$ L-ascorbic acid trisodium salt, $1 \%$ ITS, $1 \%$ Glutamax, $5 \mathrm{ng} / \mathrm{ml} \mathrm{HGF}, 20 \mathrm{ng} / \mathrm{ml}$ EGF, and $0.1 \mu \mathrm{M}$ Dex. The culture medium was changed every 2 days for a total period of 10-14 days. ${ }^{27}$

Clonogenic assays and clonal differentiation of hEHOs

Single-Cells of hEHOs were obtained based on the previous protocol. ${ }^{52}$ Briefly, the hEHOs were dissociated into single cells and incubated with $5 \mu \mathrm{g} / \mathrm{mL}$ propidium iodide (Sigma). The single- cell suspensions were then analyzed using a BD FACSCalibur as described in Fig. 5. Cells were sorted directly into organoid formation medium containing 5-10\% Matrigel in non-tissue culture-treated 96-well plates (Corning) at a density of 1 cell/ well. ${ }^{53}$ Cells were cultured and expanded in the organoid formation medium as described above. After expansion of a single cell-derived organoid for several passages, the cells were collected and subsequently placed into the media for hepatocytes versus cholangiocytes differentiation, by using the same methods described above.

\section{Immunostaining}

4\% PFA-fixed organoids were blocked with $10 \%$ Goat or Donkey serum and incubated with primary antibodies at $4{ }^{\circ} \mathrm{C}$ overnight. They were then incubated with secondary antibodies for $1 \mathrm{~h}$ in the dark at room temperature and counterstained with 4'6-diamidino2-phenylindole (DAPI). The images were observed using a fluorescence confocal microscope (Nikon TiE-A1) and were captured using NIS-Elements AR 4.40.00. For paraffin-embedded tissues or cell aggregates, $4 \mu \mathrm{m}$ sections of samples were deparaffinized, and the treatment of antigens retrieved was performed by microwaving for $10 \mathrm{~min}$. Then the sections were blocked with hydrogen peroxide and permeabilized with $0.25 \%$ Triton X-100. The subsequent steps were performed by using Vector kit (Vector Laboratories) according to the manufacturer's instructions. The images were performed using Vectra 3.0.5 and processed with the Inform 2.2.0 (PerkinElmer). A complete list of primary and secondary antibodies used was provided in Supplementary information Table S1.

\section{Quantitative real-time $P C R$}

Total RNA was prepared using RNeasy Micro or Mini Extraction Kit (Qiagen). 1 ug RNA was reverse-transcribed into CDNA using ReverTra Ace qPCR RT master mix (Toyobo, Japan). The qPCR was performed on a Bio-Rad iQ5 System using the SYBR Green PCR Master Mix (Toyobo, Japan). Expression levels were normalized to the GAPDH. A complete list of the primers used is provided in Supplementary information Table S2.

\section{Transmission electron microscopy}

The organoids samples were fixed by $2.5 \%$ glutaraldehyde at $4{ }^{\circ} \mathrm{C}$ overnight and post-fixed by incubation for $2 \mathrm{~h}$ with $1 \%$ osmium tetroxide and dehydrated in a graded series of acetone solutions. The cells were embedded in Polybed 812 epoxy resin (Polysciences, Inc, Warrington, PA). Ultrathin sections were cut and collected on 50 mesh copper grids, stained with $4 \%$ aqueous uranyl acetate for $15 \mathrm{~min}$, and then with Reynolds'lead citrate for $7 \mathrm{~min}$. Strained sections were examined with a JEM-1400 Plus electron microscope (JEOL).

Hepatocytes functional assays

To assess glycogen storage, induced hepatocyte aggregates were stained by periodic acid-Schiff (Sigma), following the manufacturer's instructions. Indocyanin green (ICG) uptake assay was performed by adding $1 \mathrm{mg} / \mathrm{ml}$ ICG (Sigma) in the medium for $30 \mathrm{~min}$. Cell uptake images were captured using an inverted microscope (Leica). ICG was completely released from the cells after about $7 \mathrm{~h}$. To assess bile canaliculi functional polarization, cells were incubated with $2 \mu \mathrm{M} 5(6)$-carboxy-2', $7^{\prime}$-dichlorofluorescein diacetate (CDFDA, Invitrogen) for $30 \mathrm{~min}$ at $37^{\circ} \mathrm{C}$. Then the culture medium was immediately discarded and the cells were washed 3 times with ice-old PBS. The image was performed on a Nikon TiE-A1 microscope.

To determine Alb secretion, the cells were cultured in the medium without phenol red, and the supernatant was collected after $24 \mathrm{~h}$. Human albumin content in the supernatant was determined using the Human Albumin ELISA kit (Bethyl Laboratory), following the manufacturer's instructions. 
To detect urea synthesis, culture medium was incubated with $10 \mathrm{mM}$ ammonium chloride (Sigma). After $24 \mathrm{~h}$, the medium was collected and QuantiChrom Urea Assay Kit (BioAssay Systems) was used according the manufacturer's instructions.

CYP3A4 activity was evaluated using P450-Glo TM CYP3A4 Luciferin-IPA kit (Promega). Briefly, cells were cultured with luminogenic substrate for $60 \mathrm{~min}$, and the supernatant was determined for luminescence (EnSight ${ }^{\mathrm{TM}} \mathrm{HH} 3400$, PerkinElmer).

CYP2E1 activity was measured according to the procedure described previously. ${ }^{54}$ CYP2E1 enzymatic activity was evaluated by the conversion of 7-methoxy-4-trifluoromethylcoumarin (MFC) to HFC. MFC (Abcam) at $10 \mu \mathrm{M}$ was added to the culture medium and incubated for $1 \mathrm{~h}$ at $37^{\circ} \mathrm{C}$. A $75 \mu \mathrm{L}$ culture supernatant was collected and mixed with $15 \mu \mathrm{L} \beta$-glucuronidase/arylsulfatase (Roche) (freshly diluted 1:100 with distilled water) and $10 \mu \mathrm{L}$ $0.5 \mathrm{M}$ sodium acetate (Sigma), and incubated for $2 \mathrm{~h}$ at $37^{\circ} \mathrm{C}$. The reaction was terminated with $100 \mu \mathrm{L} 0.25 \mathrm{~N}$ Tris in $60 \%$ acetonitrile (Sigma). The fluorescence of HFC formation was determined by EnSight $^{\mathrm{TM}}$ at Ex410/EM510. Fluorescence intensity was used to reflect the CYP2E1 activity.

Levels of liver damage enzymes ALT, AST and LDH in cell culture supernatants or in the serum of surviving hEHO- or $\mathrm{PHH}-$ transplanted FRG mice were measured using a biochemical analyzer (Photometer L100, ROBERT RIELE).

\section{Rhodamine 123 transport assay}

Cholangiocytes were incubated with $100 \mu \mathrm{M}$ rhodamine 123 (Sigma) for $5 \mathrm{~min}$ at $37^{\circ} \mathrm{C}$ and washed three times with PBS. To evaluate transport of rhodamine 123 for activity of Multidrug Resistance Protein 1 (MDR1), the cells were incubated with $10 \mu \mathrm{M}$ Verapamil (Sigma), an $\mathrm{mdr}$ inhibitor, at $37^{\circ} \mathrm{C}$ for $30 \mathrm{~min}$ before adding rhodamine 123 . The images were captured by a fluorescence microscope (Nikon Eclipse Ti-E) and analyzed with Volocity Software (PerkinElmer).

Alkaline phosphatase staining

Alkaline phosphatase staining was performed by using ALP kit (G1480, Beijing Solarbio \& Technology Co., Ltd) according to the manufacturer's instructions.

Multiparametric High-content Imaging and Analysis

The organoids were washed and stained with the selected probes using previously described methods. ${ }^{39}$ Briefly, Calcein AM and ethidium homodimer-1 (EthD-1) from LIVE/DEAD ${ }^{\text {TM }}$ Viability/ Cytotoxicity Kit were used to detect cell viability. Calcein AM and EthD-1 were used at concentration of 8 and $4 \mu \mathrm{M}$, respectively. The organoids were incubated for $30 \mathrm{~min}$ at $37^{\circ} \mathrm{C}$ and the average fluorescence intensity (AFI) of live and dead cells in the whole region was calculated for cell viability assessment based on EthD-1/ Calcein signal. Nile Red was used to localize and quantitate lipids to indicate steatosis. The organoids were incubated with $1 \mu \mathrm{M}$ Nile Red fluorescence probe for $30 \mathrm{~min}$ at $37^{\circ} \mathrm{C}$, and then were washed three times with PBS. AFI of Nile Red in the lipid spots region in cells has been used to indicate cell steatosis. CellROX ${ }^{\mathrm{TM}}$ Deep Red Reagent (CellROX) and Monochlorobimane $(\mathrm{mBCl})$ were used to quantify the reactive oxygen species (ROS) and glutathione (GSH) in cells, which represent the status of oxidative stress. The organoids were incubated with $5 \mu \mathrm{M}$ CellROX and $100 \mu \mathrm{M} \mathrm{mBCl}$ for $30 \mathrm{~min}$ at $37^{\circ} \mathrm{C}$, then washed three times with PBS. AFIs of CellROX and $\mathrm{mBCl}$ in the whole region have been used to indicate cell oxidative stress. MitoProbe $^{\mathrm{TM}} \mathrm{JC}-1$ Assay Kit (JC-1) was used to quantify alternations in mitochondrial membrane potential (MMP), which indicates damage to mitochondria. The organoids were incubated with $15 \mu \mathrm{M} \mathrm{JC}-1$ for $30 \mathrm{~min}$ in the $37^{\circ} \mathrm{C}$ incubator, then washed three times with PBS. The ratio of JC-1 red to green AFI in the whole region (JC-1 red/green ratio) has been used for mitochondria damage indication. All of the fluorescence probes used in this study were purchased from Molecular probes (Thermo Fisher Scientific). The images were acquired using an Operetta HighContent Imaging System (PerkinElmer), and saved for automated quantitative analysis using Harmony 4.1 High Content Imaging and Analysis Software.

Sirius red staining

Organoids were fixed with 4\% PFA, dehydrated in ethanol and xylene, and embedded in paraffin. The $4 \mu \mathrm{m}$ sections were deparaffinized, then stained with Sirius red (LEAGENE) for $60 \mathrm{~min}$ at room temperature, washed in the running water. They were dehydrated with two changes of $100 \%$ ethanol, and cleared in xylene. The images were prepared using Vectra 3.0.5 and processed using the Inform 2.2.0 (PerkinElmer).

\section{Measurement of pro-collagen type I secretion}

Cell culture supernatants were collected and stored at $-80^{\circ} \mathrm{C}$ until assayed. The supernatants were concentrated using $10 \mathrm{kD}$ Amicon Ultra-15 filtration columns (Millipore) at $5000 \mathrm{~g}, 15 \mathrm{~min}$. Presence of pro-collagen type I was detected by Pro-collagen Type I C-peptide ELISA kit (Takara).

mRNA sequencing and analysis

Total RNA was extracted using Trizol according to the manufacturer's instructions. RNA sequencing libraries were generated using NEBNext ${ }^{\oplus}$ Ultra $^{\mathrm{T} M}$ RNA Library Prep Kit for Illumina ${ }^{\oplus}$ (NEB, USA). Sequencing was performed by Annoroad (China). The primed 150-bp paired-end libraries were sequenced on Noves6000 platform. The reads were mapped to the human reference genome (hg21) using HISAT2. Reads Counts for each gene in each sample were counted by HTSeqv0.6.0, and FPKM (Fragments Per Kilobase Millon Mapped Reads) and then were calculated to estimate the expression level of genes in each sample. Differentially expressed genes (DEGs) were analyzed by DESeq2 using counts. Genes with $\mathrm{q} \leq 0.05$ and $\mid \log 2$ ratio $\mid \geq 1$ are identified as DEGs. Heatmap generation was performed with $\mathrm{R}$ package. Principal component analysis was performed with $\mathrm{R}$ package. Correlation analysis was performed with Corrplot package. Original data were uploaded to the Gene Expression Omnibus database (accession number GSE128717).

Cytokine array and secretome analysis

The cell culture supernatants were collected from the $\mathrm{EtOH}$ treated hFLMC/hEHO group and the EtOH-untreated hFLMC/hEHO group at day 7 of treatments. The cytokine array experiments were carried out using the RayBio Human Inflammation Antibody Array G-Series 3 (Ray Biotech, Inc.) at CapitalBio Corporation (Beijing, $\mathrm{CN}$ ). The signals from G-Series arrays were detected using an InnoScan 300 Microarray scanner (Innopsys).

Measurement of intracellular calcium

$\mathrm{hEHO}-\mathrm{Clcs}$ were incubated with $25 \mu \mathrm{M}$ of the calcium indicator Fluo-4 a.m. (Life Technologies) for $60 \mathrm{~min}$ at $37^{\circ} \mathrm{C}$ and washed 3 times using Williams' E medium. hEHO-Clcs were stimulated with $1 \mu \mathrm{M}$ acetylcholine and $30 \mu \mathrm{M}$ ATP (Sigma). The time-lapse cell images were captured by inverted fluorescence microscope (Nikon Eclipse Ti-E). The images were processed with Volocity Software. Each experiment was repeated in triplicate.

Isolation of primary human cholangiocytes from liver tissue Human hepatocytes were isolated from liver tissue by a modified two-step collagenase perfusion technique as described above. Isolated cells were centrifuged for $3 \mathrm{~min}$ at $75 \mathrm{~g}$. Supernatant was collected and spun at $300-400 \mathrm{~g}$ for $5 \mathrm{~min}$. Cell pellets were washed three times in cold DMEM/F12 medium and labeled with PE-CF594 Mouse Anti-Human CD326 (EpCAM, BD Biosciences) for $30 \mathrm{~min}$ at $4{ }^{\circ} \mathrm{C}$. The stained cells were washed and sorted with $\mathrm{BD}$ FACSCalibur. 
Statistical analysis

Statistical analysis was performed using GraphPad Prism 5 (GraphPad Software Inc., La Jolla, CA, USA). Data were shown as mean \pm standard deviation (SD). Two-tailed Student's t test was used and $P$ value $<0.05$ was considered statistically significant. For all statistics, data from at least three experiments were used.

\section{ACKNOWLEDGEMENTS}

We thank Drs Zhigui Zeng, and Zhijun Zhu for their help with clinical samples; Drs Lola Reid and Xin Wang for critical review. Dr Xin Chang for TEM experiments; $\mathrm{Mr}$ Zhimin $\mathrm{Li}$ and Chuanwen Wang for bioinformatics analysis. This work was supported by the National Natural Science Foundations of China (No. 81730052), the Interdisciplinary Cooperation Project of Beijing Nova Program (Z1811100006218127), the National Major Scientific and Technological Special Project for "Significant New Drugs Development" (2018ZX09711003-001-002), the National Key Research and Development Program of China (No. 2016YFC1101305), the Science and Technology Planning Project of Guangdong China (2015A050502023), the Guangdong Province Science and Technology Program (2018KJYZ021) and Science and Technology Program of Guangzhou, China (STPG; 2016201604030054).

\section{AUTHOR CONTRIBUTIONS}

Y.W. and S.W. conceived and designed the project. S.W. and X.W. conducted most of the experiments. Y.W., S.W., and X.W. wrote and edited the manuscript. Z.T., Y.S., and M.C. contributed to studies with cell culture, IF and tissue histology. J.L. helped with HCA experiments. F.Y. helped with in vivo transplantation experiments. J.C., T.C., C.L., and J.H. reviewed the final version of the manuscript.

\section{ADDITIONAL INFORMATION}

Supplementary information accompanies this paper at https://doi.org/10.1038/ s41422-019-0242-8.

Competing interests: The authors declare no competing interests.

\section{REFERENCES}

1. Clevers, H. Modeling development and disease with organoids. Cell. 165 1586-1597 (2016).

2. Sato, T. et al. Single Lgr5 stem cells build crypt-villus structures in vitro without a mesenchymal niche. Nature. 459, 262 (2009).

3. Eiraku, M. et al. Self-organizing optic-cup morphogenesis in three-dimensional culture. Nature. 472, 51 (2011).

4. Lancaster, M. A. et al. Cerebral organoids model human brain development and microcephaly. Nature. 501, 373 (2013).

5. Takasato, M. et al. Kidney organoids from human iPS cells contain multiple lineages and model human nephrogenesis. Nature. 536, 238 (2016).

6. Huch, M. et al. Unlimited in vitro expansion of adult bi-potent pancreas progenitors through the Lgr5/R-spondin axis. EMBO J. 32, 2708-2721 (2013).

7. Yamamoto, Y. et al. Long-term expansion of alveolar stem cells derived from human iPS cells in organoids. Nat. Methods. 14, 1097 (2017).

8. Boj Sylvia F. et al. Organoid models of human and mouse ductal pancreatic cancer. Cell. 160, 324-338 (2015).

9. Huch, M. et al. Long-term culture of genome-stable bipotent stem cells from adult human liver. Cell. 160, 299-312 (2015).

10. $\mathrm{Hu}, \mathrm{H}$. et al. Long-term expansion of functional mouse and human hepatocytes as 3D organoids. Cell. 175, 1591-1606 e1519 (2018).

11. Ogawa, S. et al. Three-dimensional culture and CAMP signaling promote the maturation of human pluripotent stem cell-derived hepatocytes. Development. 140, 3285-3296 (2013).

12. Gieseck, R. L. 3rd et al. Maturation of induced pluripotent stem cell derived hepatocytes by 3D-culture. PloS one. 9, e86372-e86372 (2014).

13. Rashidi, H. et al. 3D human liver tissue from pluripotent stem cells displays stable phenotype in vitro and supports compromised liver function in vivo. Arch. Toxicol. 92, 3117-3129 (2018).

14. Takebe, T. et al. Vascularized and functional human liver from an iPSC-derived organ bud transplant. Nature. 499, 481-484 (2013).

15. Guan Y., et al. Human hepatic organoids for the analysis of human genetic diseases. JCl Insight. 2, e94954 (2017).

16. Touboul, T. et al. Generation of functional hepatocytes from human embryonic stem cells under chemically defined conditions that recapitulate liver development. Hepatology. 51, 1754-1765 (2010).
17. Si-Tayeb, K. et al. Highly efficient generation of human hepatocyte-like cells from induced pluripotent stem cells. Hepatology. 51, 297-305 (2010).

18. Baxter, M. et al. Phenotypic and functional analyses show stem cell-derived hepatocyte-like cells better mimic fetal rather than adult hepatocytes. J. Hepatol. 62, 581-589 (2015).

19. Qin, J. et al. Connexin 32-mediated cell-cell communication is essential for hepatic differentiation from human embryonic stem cells. Sci. Rep. 6, 37388 (2016).

20. Huch, M. et al. In vitro expansion of single Lgr5+ liver stem cells induced by Wntdriven regeneration. Nature. 494, 247-250 (2013).

21. Broutier, L. et al. Human primary liver cancer-derived organoid cultures for disease modeling and drug screening. Nat. Med. 23, 1424-1435 (2017).

22. Horslen, S. P. \& Fox, I. J. Hepatocyte transplantation. Transplantation. 77, 1481-1486 (2004).

23. Hannan, N. R. et al. Generation of multipotent foregut stem cells from human pluripotent stem cells. Stem Cell Rep. 1, 293-306 (2013).

24. Ogawa, M. et al. Directed differentiation of cholangiocytes from human pluripotent stem cells. Nat. Biotechnol. 33, 853-861 (2015).

25. Avior, Y. et al. Microbial-derived lithocholic acid and vitamin K2 drive the metabolic maturation of pluripotent stem cells-derived and fetal hepatocytes. Hepatology. 62, 265-278 (2015).

26. Tanimizu, N., Miyajima, A. \& Mostov, K. E. Liver progenitor cells develop cholangiocyte-type epithelial polarity in three-dimensional culture. Mol. Biol. Cell. 18, 1472-1479 (2007)

27. Sampaziotis, F. et al. Cholangiocytes derived from human induced pluripotent stem cells for disease modeling and drug validation. Nat. Biotechnol. 33, 845-852 (2015).

28. Azuma, H. et al. Robust expansion of human hepatocytes in Fah(-/-)/ Rag2(-/-)/II2rg (-/-) mice. Nat. Biotechnol. 25, 903-910 (2007).

29. Wang, Y. et al. Conversion of human gastric epithelial cells to multipotent endodermal progenitors using defined small molecules. Cell Stem Cell. 19, 449-461 (2016).

30. Yan, F. et al. Human embryonic stem cell-derived hepatoblasts are an optimal lineage stage for hepatitis C virus infection. Hepatology. 66, 717-735 (2017).

31. Benhamouche, S. et al. Apc tumor suppressor gene is the "zonation-keeper" of mouse liver. Dev. Cell. 10, 759-770 (2006).

32. Leite, S. B. et al. Novel human hepatic organoid model enables testing of druginduced liver fibrosis in vitro. Biomaterials. 78, 1-10 (2016).

33. Coll, M. et al. Generation of hepatic stellate cells from human pluripotent stem cells enables in vitro modeling of liver fibrosis. Cell Stem Cell. 23, 101-113 e107 (2018).

34. Si-Tayeb, K., Lemaigre, F. P. \& Duncan, S. A. Organogenesis and development of the liver. Dev. Cell. 18, 175-189 (2010).

35. Joshi, M. et al. Fetal liver-derived mesenchymal stromal cells augment engraftment of transplanted hepatocytes. Cytotherapy. 14, 657-669 (2012)

36. Henderson, N. C. et al. Targeting of av integrin identifies a core molecular pathway that regulates fibrosis in several organs. Nat. Med. 19, 1617-1624 (2013).

37. Mederacke, I. et al. Fate tracing reveals hepatic stellate cells as dominant contributors to liver fibrosis independent of its aetiology. Nat. Commun. 4, 2823 (2013).

38. Louvet, A. \& Mathurin, P. Alcoholic liver disease: mechanisms of injury and targeted treatment. Nat. Rev. Gastroenterol. Hepatol. 12, 231-242 (2015).

39. Liu J., et al. Liver extracellular matrices bioactivated hepatic spheroids as a model system for drug hepatotoxicity evaluations. Adv Biosyst 2, 1800110 (2018).

40. King, A. L. et al. Involvement of the mitochondrial permeability transition pore in chronic ethanol-mediated liver injury in mice. Am. J. Physiol. Gastrointest. Liver Physiol. 306, G265-G277 (2014).

41. Osna, N. A., Donohue, T. M. \& Kharbanda, K. K. Alcoholic liver disease: pathogenesis and current management. Alcohol Res. 38, 147-161 (2017).

42. Xu, M. J., Zhou, Z., Parker, R. \& Gao, B. Targeting inflammation for the treatment of alcoholic liver disease. Pharm. Ther. 180, 77-89 (2017).

43. Petrasek, J. et al. IL-1 receptor antagonist ameliorates inflammasome-dependent alcoholic steatohepatitis in mice. J. Clin. Investig. 122, 3476-3489 (2012).

44. Tilg, H., Moschen, A. R. \& Szabo, G. Interleukin-1 and inflammasomes in alcoholic liver disease/acute alcoholic hepatitis and nonalcoholic fatty liver disease/nonalcoholic steatohepatitis. Hepatology. 64, 955-965 (2016).

45. Lemmers, A. et al. The interleukin-17 pathway is involved in human alcoholic liver disease. Hepatology. 49, 646-657 (2009).

46. Takebe, $T$. et al. Massive and reproducible production of liver buds entirely from human pluripotent stem cells. Cell Rep. 21, 2661-2670 (2017).

47. Avior, Y. et al. Microbial-derived lithocholic acid and vitamin K2 drive the metabolic maturation of pluripotent stem cells-derived and fetal hepatocytes. Hepatology. 62, 265-278 (2015).

48. Dolganiuc, A. \& Szabo, G. In vitro and in vivo models of acute alcohol exposure World J. Gastroenterol. 15, 1168-1177 (2009). 
49. Jones, C. N. et al. Cultivating hepatocytes on printed arrays of HGF and BMP7 to characterize protective effects of these growth factors during in vitro alcohol injury. Biomaterials. 31, 5936-5944 (2010).

50. Lecluyse, E. L. \& Alexandre, E. Isolation and culture of primary hepatocytes from resected human liver tissue. Methods Mol. Biol. 640, 57-82 (2010).

51. Broutier, L. et al. Culture and establishment of self-renewing human and mouse adult liver and pancreas 3D organoids and their genetic manipulation. Nat. Protoc. 11, 1724 (2016).
52. Katsuda, T. et al. Conversion of terminally committed hepatocytes to culturable bipotent progenitor cells with regenerative capacity. Cell Stem Cell. 20, 41-55 (2017).

53. Li, B. et al. Adult mouse liver contains two distinct populations of cholangiocytes. Stem Cell Rep. 9, 478-489 (2017).

54. Donato, M. T., Jiménez, N., Castell, J. V. \& Gómez-Lechón, M. J. Fluorescencebased assays for screening nine cytochrome P450 (P450) activities in intact cells expressing individual human P450 enzymes. Drug Metab. Dispos. 32, 699 (2004). 